This item was submitted to Loughborough's Research Repository by the author.

Items in Figshare are protected by copyright, with all rights reserved, unless otherwise indicated.

\title{
Short-lived large-amplitude pulses in the nonlinear long-wave model described by the modified Korteweg-de Vries equation
}

PLEASE CITE THE PUBLISHED VERSION

LICENCE

CC BY-NC-ND 4.0

\section{REPOSITORY RECORD}

Grimshaw, Roger H.J., Efim N. Pelinovsky, Tatiana G. Talipova, Michael Ruderman, and Robert Erdelyi. 2019. "Short-lived Large-amplitude Pulses in the Nonlinear Long-wave Model Described by the Modified Kortewegde Vries Equation”. figshare. https://hdl.handle.net/2134/241. 


\title{
Short-lived large-amplitude pulses in the nonlinear long-wave model
}

\section{described by the modified Korteweg-de Vries equation}

\author{
Roger Grimshaw ${ }^{1)}$, Efim Pelinovsky ${ }^{2)}$, Tatiana Talipova ${ }^{2)}$, Michael Ruderman ${ }^{3)}$, and
}

Robert Erdelyi ${ }^{3)}$,

\footnotetext{
${ }^{1)}$ Department of Mathematical Sciences, Loughborough University, Loughborough, UK

${ }^{2)}$ Laboratory of Hydrophysics and Nonlinear Acoustics, Institute of Applied Physics, Nizhny Novgorod, Russia

${ }^{3)}$ Department of Applied Mathematics, University of Sheffield, Sheffield, UK
}

Version 3 March 262004

\begin{abstract}
:
The appearance and disappearance of short-lived large-amplitude pulses in a nonlinear long wave model is studied in the framework of the modified Korteweg-de Vries equation. The major mechanism of such wave generation is modulational instability leading to the generation and interaction of the breathers. The properties of breathers are studied both within the modified Korteweg -de Vries equation, and also within the nonlinear Schrödinger equation derived by an asymptotic reduction from the modified Korteweg -de Vries for weakly nonlinear wave packets, The associated spectral problems (AKNS or Zakharov-Shabat) of the inverse-scattering transform technique also utilized. Wave formation due to this modulational instability is investigated for localized and for periodic disturbances. Nonlinear-dispersive focusing is identified as a possible mechanism for the formation of anomalously large pulses.
\end{abstract}




\section{Introduction}

The modified Korteweg -de Vries (mKdV) equation

$$
\frac{\partial u}{\partial t}+6 u^{2} \frac{\partial u}{\partial x}+\frac{\partial^{3} u}{\partial x^{3}}=0
$$

is a well-known canonical model nonlinear wave equation in mathematical physics. It can be solved exactly by the inverse-scattering transform method for either sign of the coefficient of the nonlinear term in (1) (Lamb, 1980; Ablowitz and Clarkson, 1991). This equation with a positive coefficient as in (1) appears in applications to various physical problems: meandering barotropic jets (Nycander et al, 1993; Ralph and Pratt, 1994), internal waves in stratified fluids (Grimshaw et al, 1997; Talipova et al, 1999), nonlinear Alfvén waves propagating along the ambient magnetic field (Kakutani and Ono, 1969; Mjølhus, 1989; Mjølhus and Hada, 1997), and nonlinear waves in a distributed Schottky barrier diode transmission lines (Zieger et al, 2001). The "positive" modified Korteweg-de Vries equation has elementary solutions (see, for instance, Lamb, 1980) in the form of solitary waves of either polarity (that is, $a$ the soliton amplitude can take either sign)

$$
u(x, t)=a \operatorname{sech}\left[a\left(x-a^{2} t\right)\right]
$$

and breathers (oscillatory wave packets)

$$
\begin{gathered}
u(x, t)=-4 q \operatorname{sech} \theta\left[\frac{\cos \phi-(q / p) \sin \phi \tanh \theta}{1+(q / p)^{2} \sin ^{2} \phi \cdot \operatorname{sech}^{2} \theta}\right], \\
\phi=2 p x+8 p\left(p^{2}-3 q^{2}\right) t, \quad \theta=2 q x+8 q\left(3 p^{2}-q^{2}\right) t .
\end{gathered}
$$

There are also singular solutions (positons, compacton-like and solitary patterns-like solutions, see Matveev, 2002, Wazwaz, 2003) but their physical meaning is not evident.

Recently, Clarke et al (2000) solved the Cauchy problem for the modified Korteweg-de Vries (1) for the localized disturbances (that is, disturbances vanishing at infinity) and determined conditions for the formation of solitons (2) and breathers (3). The dynamics of almost periodic perturbations has received much less attention. Among the results concerning periodic and 
almost periodic initial perturbations we can mention the study of modulational instability of periodic (“cnoidal”) waves by Driscoll and O'Neil (1976). They showed that periodic waves with a zero mean value (that is, no pedestal) are unstable with respect to long-wave modulations. The parameters of this instability (the increment and range of wavenumbers) were calculated in the small-amplitude approximation. These results for small-amplitude waves have been confirmed in the framework of the nonlinear Schrodinger equation derived by asymptotic reduction from the modified Korteweg -de Vries equation (Parkes, 1987; Grimshaw et al, 2001). It is important to point out that the nonlinear Schrodinger equation is itself a very well-studied nonlinear evolution equation. An important result in the framework of this equation is that the evolution of a weakly modulated wavetrain may lead to the generation of short-lived largeamplitude pulses called in oceanic applications, freak or rogue waves (Dysthe and Trulsen, 1999; Osborne al, 2000; Ablowitz et al, 2001; Kharif et al, 2001; Calini and Schober, 2002; Slunyaev et al, 2002; Kharif and Pelinovsky, 2003). The main aim of this present study is to analyze the unsteady wave dynamics and anomalous wave behaviour in the framework of the modified Korteweg-de Vries equation (1) for a wide range of the wave parameters, and to compare it with the corresponding predictions of the weakly nonlinear model based on the nonlinear Schrodinger equation.

\section{Theoretical background}

According to the solution of the initial-value problem for the modified Korteweg-de Vries equation (1) obtained from the inverse-scattering transform method, any localized initial disturbance of finite energy (that is, vanishes sufficiently fast at infinity) evolves into a set of solitons, breathers and a dispersive wave train. The solitons and breathers can be obtained through the Ablowitz-Kaup-Newell-Segur (AKNS) or Zakharov-Shabat schemes (Lamb, 1980; Ablowitz and Clarkson, 1991)

$$
\begin{aligned}
& \frac{\partial \varphi_{1}}{\partial x}=-u(x) \varphi_{2}+\lambda \varphi_{1}, \\
& \frac{\partial \varphi_{2}}{\partial x}=+u(x) \varphi_{1}-\lambda \varphi_{2},
\end{aligned}
$$


where $u(x)$ is an initial disturbance, and $\lambda$ is a complex-valued eigenvalue. If the initial disturbance vanishes rapidly at infinity, the spectrum of this associated problem (4) can contain discrete eigenvalues. A real eigenvalue corresponds to the solitary wave (2) with amplitude $a=$ $2 \lambda$, while the complex discrete eigenvalue, $\lambda=q \pm i p$, corresponds to a breather (3). A detailed investigation of the generation of solitons and breathers from various piecewise-constant localized initial disturbances has been carried out by Clarke et al (2000). The solitons and breathers generated from such localized disturbances have different velocities and the initial disturbance fissions into separated waves. Note that the interaction of solitons and breathers is elastic, so that they "push off" each other. As a result, they cannot form groups, and so induce the appearance of very large-amplitude pulses (freak waves) due to the collision of solitons and breathers.

If an initial disturbance is wide enough, or does not vanish at infinity, the character of the wave dynamics is more complicated. In the particular case of periodic boundary conditions the elementary solution of the modified Korteweg -de Vries equation (1) is the cnoidal wave

$$
u(x, t)=k \operatorname{sen}(\omega t-k x, s), \quad \omega=-k^{3}\left(1-2 s^{2}\right),
$$

where $\operatorname{cn}(\theta, s)$ is the Jacobi elliptic function with the modulus $s(0<s<1)$. In the approximation of weak nonlinearity $(s<<1)$ the wave shape is sinusoidal and the wave propagates to the left, while strongly nonlinear waves $(s \rightarrow 1)$ are similar to a lattice of solitons of opposite polarities and propagate to the right. For the domain of length $L$ with periodic boundary conditions (as is typical in numerical simulations), the wave number, $k$, and module, $s$, are related by

$$
k_{n} L=4 n K(s) \text {, }
$$

where $K(s)$ is the elliptic integral of the first kind. The wave amplitude is determined by the module s, which is a free parameter.

Using the inverse-scattering transform technique (or the Hirota bilinear method) the nonlinear superposition of cnoidal waves (the so called $N$-degree of freedom cnoidal wave solutions) can also be obtained. These solutions correspond to a multi-zonal potential in the associated spectral problem (4). Importantly for applications the cnoidal wave is unstable with respect to long- 
wave modulations (Driscoll and O’Neil, 1976). This result holds for the cnoidal wave (5) for any value of the modulus $s$. However, the nonlinear regime of this modulational instability of cnoidal waves has not been investigated.

The behavior of weakly nonlinear packets can be analyzed in detail using the nonlinear Schrodinger equation (NLS), which can be derived by asymptotic reduction from the modified Korteweg-de Vries equation (Lamb, 1980; Parkes, 1987; Grimshaw et al, 2001). Thus, on representing the wave field as the Fourier series

$$
u(x, t)=\varepsilon A(X, T) \exp (i k x-\omega t)+c . c+\varepsilon^{2} \ldots,
$$

where

$$
\omega=-k^{3}, \quad X=\frac{x-c_{g r} t}{\varepsilon}, \quad T=\frac{3 k t}{\varepsilon^{2}}, \quad c_{g r}=\frac{d \omega}{d k}=-3 k^{2}, \quad \varepsilon<<1,
$$

and using the well-known asymptotic procedure, the following equation for the complex envelope can be derived at the leading order (Lamb, 1980; Parkes, 1987; Grimshaw et al, 2001)

$$
i \frac{\partial A}{\partial T}=\frac{\partial^{2} A}{\partial X^{2}}+2|A|^{2} A
$$

In fact, the small parameter, $\varepsilon$ characterizes the ratio between the envelope wavenumber, $K$ and the carrier wavenumber $k$, so that $\varepsilon=K / k$. Also, we should mention that the amplitude modulation of the wave field (due to its reality) is

$$
a(X, T)=2|A(X, T)|
$$

The NLS equation is also a canonical model nonlinear wave equation in mathematical physics, and it has been studied in great detail. For a positive coefficient of the nonlinear term in the modified Korteweg-de Vries equation, the NLS equation (8) is the "focusing" nonlinear Schrodinger equation. In this case a monochromatic wave of amplitude $A_{0}$ is unstable with respect to a long-wavelength envelope perturbation with the wave numbers $\Delta k$ satisfying 


$$
\Delta k<K_{\max }=2 A_{0} .
$$

The maximum growth rate of this instability is

$$
\gamma=2 A_{0}^{2}
$$

and it is attained for $\Delta k=K_{\max } / \sqrt{ } 2$. The existence of unstable modes in the NLS equation results in very complicated nonlinear dynamics of wave packets (see Osborne al, 2000; Ablowitz et al, 2001; Calini and Schober, 2002). It is important to note that the nonlinear Schrodinger equation has almost the same associated spectral problem (4) as the modified Korteweg-de Vries equation (Lamb, 1980; Ablowitz and Clarkson, 1991),

$$
\begin{aligned}
& \frac{\partial \psi_{1}}{\partial x}=-A(x) \psi_{2}+\mu \psi_{1}, \\
& \frac{\partial \psi_{2}}{\partial x}=+A^{*}(x) \psi_{1}-\mu \psi_{2},
\end{aligned}
$$

and in the case of the initial "pure amplitude" modulated $\left(A=A^{*}\right)$ packets the system (12) coincides in form with (4). The system (12) can be asymptotically derived directly from the AKNS system (4) after the substitution (7) at $t=0$ and representing the eigenfunctions of (4) as the asymptotic expansion,

$$
\varphi_{1}=\psi_{1}(X) e^{\lambda_{0} x}+\varepsilon \psi_{1}^{(1)}+\ldots ., \quad \varphi_{2}=\psi_{2}(X) e^{-\lambda_{0} x}+\varepsilon \psi_{2}^{(1)}+\ldots, \quad \lambda=\lambda_{0}+\varepsilon \mu+\ldots .
$$

Substitution into (4) and removal of secular terms at order $\varepsilon$ in the equations for $\psi^{(l)}$ easily give that $\lambda_{0}= \pm i k / 2$ and that $\psi_{1,2}$ satisfy (12). Thus, any eigenvalue $\mu$ of (12) (real-valued for a "pure" amplitude modulation) giving an envelope soliton for the nonlinear Schrodinger equation corresponds to a complex eigenvalue asymptotically given by $\mu \pm i k / 2$ for (4), which corresponds to a breather. This agrees with the direct breather reduction from the $\mathrm{mKdV}$ equation to the NLS equation (Lamb, 1980; Grimshaw et al, 2001). 
For disturbances that tend to a constant at infinity (that is, they exist on a pedestal), a single discrete eigenvalue $\lambda=\cos (\eta+i \phi)$ corresponds to the breather solution of the nonlinear Schrodinger equation (see, e.g., Dysthe and Trulsen, 1999)

$$
A(x, t)=\exp (-2 i t) \frac{\cos (2 \gamma t+2 i \phi)-\cosh \phi \cosh (2 \Gamma x)}{\cos (2 \gamma t)-\cosh \phi \cosh (2 \Gamma x))}, \quad \Gamma=\sinh \phi, \quad \gamma=\sinh (2 \phi),
$$

where $\phi$ is a free parameter. This breather is localized in space and periodic in time. The important role of the breather solutions in freak wave formation in the framework of the nonlinear Schrodinger equation has been pointed out by Dysthe and Trulsen (1999). These authors also presented other breather solutions. Physically such solutions demonstrate the formation of short-lived energetic wave packets from an almost periodic wavetrain in the framework of the $\mathrm{mKdV}$ equation. The height of these anomalous waves can exceed the unperturbed value of the wavetrain by up to three times. Such waves can appear at any place and at any time and, in contrast to solitons and breathers, they exist only for finite periods of time. As a result, an initial quasi-uniform wave field will evolve into one with a variable number of chaotically appearing and disappearing large-amplitude wave packets.

\section{Breather generation from localized wave packets}

First, the dynamics of weakly nonlinear wave packets in the framework of the modified Korteweg -de Vries and NLS equations will be considered. The initial wave group is chosen as

$$
u(x)=\left\{\begin{array}{lc}
0 & x<0, \\
a \sin (k x) & 0<x<2 \pi, \\
0 & x>2 \pi,
\end{array}\right.
$$

where the wavenumber and amplitude are varied ( $k$ is an integer). Accordingly, the wave envelope, $A(x)$ in the nonlinear Schrodinger equation is described by

$$
A(x)= \begin{cases}0 & x<0 \\ a / 2 & 0<x<2 \pi \\ 0 & x>2 \pi\end{cases}
$$


The advantage of the chosen piece-constant envelope is that the AKNS system for the modified Korteweg - de Vries equation (4) as well as (12) for the NLS equation can be solved in the finite domain $2 \pi$, with the boundary conditions (see for instance, Clarke et al (2000)),

$$
\varphi_{1} \text { and } \psi_{1}=0 \text { at } x=2 \pi, \quad \text { and } \quad \varphi_{2} \text { and } \psi_{2}=0 \text { at } x=0 .
$$

The solutions of the both AKNS systems are can then be found explicitly, and the discrete spectra determined.

The first run is done for the slowly modulated wave packets: $\boldsymbol{k}=\mathbf{1 0}$ and $\boldsymbol{a}=\mathbf{2}$. Two discrete eigenvalues are obtained for the initial modified Korteweg-de Vries equation

$$
\begin{aligned}
& \lambda_{1}=(0.54 \pm 0.004) \pm(4.95 \pm 0.02) i, \\
& \lambda_{2}=(0.90 \pm 0.004) \pm(4.95 \pm 0.02) i,
\end{aligned}
$$

and for the nonlinear Schrodinger equation

$$
\begin{aligned}
& \mu_{1}=0.538 \pm 0.004 \\
& \mu_{2}=0.904 \pm 0.004
\end{aligned}
$$

As expected in this weakly nonlinear limit, the imaginary part of the eigenvalues is close to $\operatorname{Im}(\lambda)=k / 2=5$, and the real part of the eigenvalues are approximately the same: $\operatorname{Re}(\lambda)=\mu$. Therefore, two breathers are generated from the initial wave field in the framework of the modified Korteweg-de Vries equation, and they are envelope solitons in the framework of the nonlinear Schrodinger equation. It is important to mention that characteristics of the carrier wave in both breathers are the same (phase and group velocities), and therefore, their relative motion is related with nonlinearity only.

Also, we carried out direct numerical simulation of the modified Korteweg-de Vries equation as well as for the nonlinear Schrodinger equation for the corresponding initial conditions: (15) for $\mathrm{mKdV}$ and (16) for NLS. Snapshots of the wave field evolution in the framework of the mKdV equation (left) and NLS equation (right) are presented in Fig. 1 for the same times. It is clearly seen that both models give the same description of the wave packet evolution into two breathers and a weak dispersive tail. These snapshots demonstrate the appearance and disappearance of the large amplitude short-lived pulses. According to the AKNS scheme the breather amplitude is 
$4 \mu$; see (2). The breather amplitudes are 3.6 and 2.2, but it is difficult to select "pure" breathers because they oscillate around the "middle" point. The variation of maximum wave amplitude in the process of the breather interaction is large, from 2.5 up to 5.8, more than twice. Largeamplitude pulses appear for a short time and then disappear. Meanwhile, the maximum wave amplitude does not exceed the sum of breather amplitudes.

The second run is done for strongly modulated wave packets: $\boldsymbol{k}=\mathbf{4}$ and $\boldsymbol{a}=4$. The discrete spectrum for the AKNS system for the modified Korteweg-de Vries equation is

$$
\begin{aligned}
& \lambda_{1}=(0.664 \pm 0.004) \pm(0.888 \pm 0.004) i, \\
& \lambda_{2}=(1.512 \pm 0.004) \pm(1.296 \pm 0.004) i, \\
& \lambda_{3}=(1.896 \pm 0.004) \pm(1.408 \pm 0.004) i .
\end{aligned}
$$

The same calculation for the nonlinear Schrodinger equation gives

$$
\begin{aligned}
& \mu_{1}=0.824 \pm 0.004, \\
& \mu_{2}=1.444 \pm 0.004, \\
& \mu_{3}=1.770 \pm 0.004
\end{aligned}
$$

The eigenvalues in both models now differ significantly because there is no small parameter characterized the weak modulation. The snapshots of the wave field evolution computed directly in the framework of $\mathrm{mKdV}$ (left) and NLS (right) are presented in Fig. 2. First, the NLS equation overestimates the large-amplitude waves (its amplitude reaches 12 ), while the $\mathrm{mKdV}$ equation gives the maximum amplitude as 9.3. Moreover, the breathers in the NLS model oscillate near the "middle" point, and the large-amplitude pulse will appear and disappear often. In the mKdV model all breathers have different carrier wave number (see (20)), and therefore, group velocities. For large times breathers completely separate (Fig. 3) and large-amplitude pulses appear only at the beginning stage.

\section{Numerical simulation of modulated periodic wavetrains}

In this section we present the results of numerical solution of the modified Korteweg-de Vries equation (1) with periodic boundary conditions. Although the correspondence between the mkdV equation and the NLS equation still holds here for weakly nonlinear waves, the 
corresponding spectral problems need to be considered with periodic boundary conditions; this case is much more complicated and less developed than that for localized solutions, and indeed from our perception, not so easily applied, and so we shall not consider that aspect here. To analyze the modulational instability of periodic waves we have imposed the initial condition corresponding to an amplitude-modulated sinusoidal wave,

$$
u(x, 0)=a(1+m \sin (K x)) \sin (k x) .
$$

Since we use periodic boundary conditions at the domain boundaries, the ratio $k / K$ is an integer. The first run is carried out for the case when the wave is stable in the weakly nonlinear limit. The parameters are: $a=0.04, m=0.05, k=1.884$, and $K=0.157$. The wave evolution is shown on Fig. 4 for three different moments of time. As is predicted by the weakly nonlinear theory, the wave envelope demodulates and then almost returns to the initial state.

The next run is carried out for the conditions corresponding to the modulational instability (10). The parameters are: $a=0.08, m=0.05, k=1.884, K=0.00785$. The wave dynamics is displayed in Fig. 5 where the intensity corresponds to the value of $u(x, t)$. The weak modulation of the wave packet is almost invisible up to time $t=300$, and then the wave field becomes nonuniform due to modulational instability. Intense wave packets exist for times 300-900. These packets cross the domain several times due to the periodic boundary conditions. The wave field varies significantly with time forming separated "spots" shown in large scale in Fig. 6 extracted from Fig. 5 for $t<600$. Such spots correspond to the appearance and disappearance of wave packets (breather-like packets). Between times $t=450$ and $t=500$ a large-amplitude breather has crossed the domain once but disappears after that. Between times $t=600$ and $t=750$ the wave field is not very intense, excluding a small time interval $t=660-670$ when a more intense breather crosses the domain once and then disappears. The wave intensity grows at $t=750$ and continues to grow up to $t=900$. After that (up to $t=1150$ ) there is a time interval of near recurrence of the initial slowly modulated wave. In the vicinity of $t=1200$ and $t=1400$ intense wave packets appeared again. Between $t=1500$ and $t=1700$ the wave field has almost returned to the initial state. The analysis of wave trajectories allows us to select the moments when a large-amplitude pulse appears. Snapshots of the wave field are shown in Figure 7. As predicted in the framework of the NLS equation, the development of modulational instability leads to the appearance of short-lived and almost symmetric wave groups. The simulations demonstrate also the recurrence phenomenon known for integrable system (see wave profiles at $t=1038$ and $t=$ 
1578). The results of numerical simulations in the framework of the $\mathrm{mKdV}$ equation show some differences in comparison to the NLS equation. In fact, the wave profile at $t=462$ is nonsymmetric (with respect to the large-amplitude pulse), while it would be symmetric in the NLS equation if the initial condition is symmetric. The maximum of the wave amplitude (in the domain) versus time is shown in Fig. 8. It is necessary to note that the maximum value of the wave amplitude (0.31) exceeds the initial wave amplitude (0.08) by 3.8 times, while in the framework of the NLS equation the maximum amplitude of a breather does not exceed the background amplitude by more than 3 times.

The next run is carried out for a wave of moderate amplitude, $a=0.16$ (other parameters are the same: $k=1.884, m=0.05, K=0.00785)$. Wave trajectories are shown in Fig. 9. Pulses with significant amplitude start to appear at $t=100$. The life-time of these pulses is short, and it is difficult to separate different traces one from another. Pulses are interacting, and the wave amplitude varies rapidly. It is worth noting that there is no visible recurrence to the initial state. Snapshots of the wave field are presented in Fig. 10. The instability of a wave of moderate amplitude leads to the appearance of wave groups with anomalously large amplitudes. These wave groups contain different numbers of individual waves. In fact, highly energetic waves are always present in the wave field, as can be seen in Fig. 11 where the maximum wave amplitude (in the domain) versus time is shown. The maximum wave amplitude varies between 0.26 and 0.6 ; hence the maximum amplification is 1.5 , which is almost the same as for weakly nonlinear waves. Oscillations of the maximum wave amplitude occur more frequently because the characteristic time of the modulational instability decreases when the wave amplitude increases.

The last run is carried out for a wave of large amplitude $(a=0.32)$, other parameters being the same $(k=1.884, m=0.05, K=0.00785)$. The wave trajectories are shown on Figure 12 . There is a lot of spatially narrow wave packets that appear and disappear rapidly, so that their lifetime is short. Waves with very large amplitudes appear randomly as can be seen in Fig. 13 where the waves with amplitudes exceeding 0.4 are present. The irregular character of the wave field is demonstrated by the snapshots displayed in Fig. 14. Sometimes single large-amplitude pulses appear instead of wave groups. The maximum of the wave amplitudes in the domain varies from 0.6 to 1.1 (Fig. 15), and the maximum amplification is 1.4. The maximum amplification decreases when the initial wave amplitude increases. Simultaneously the variation of the maximum amplitude decreases. 


\section{Nonlinear Dispersive Focusing}

Briefly, we describe an alternative mechanism for the generation of short-lived large-amplitude pulses, in which there is a collision of dispersive, frequency modulated wave trains propagating with different group velocities. This mechanism called wave focusing is well known for linear wave packets, and also occurs for weakly nonlinear wave packets (Pelinovsky et al, 2000; Kharif et al, 2001; Slunyaev et al, 2002). Here the mechanism can be demonstrated by using the self-similar solution of the modified Korteweg-de Vries equation

$$
u(x, t) \sim \frac{1}{t^{1 / 3}} F\left(x / t^{1 / 3}\right)
$$

where $F(z)$ is the Painleve transcedents satisfying the Painleve-2 equation (Ablowitz and Clarkson, 1991)

$$
\frac{d^{2} F}{d z^{2}}+\frac{1}{3}\left(-z F+F^{3}\right)=0
$$

The behavior of the solution given by (24) is similar to the behavior of the Airy-function (note that in the linear approximation, when the cubic nonlinear term is omitted, $F$ is an Airy function). It is important to note that this self-similar solution describes the evolution of the $\delta$ function (which can be considered as a model of an anomalously large wave). Due to invariance of the modified Korteweg-de Vries equation in space and time, the solution (24) will remain a solution of the $\mathrm{mKdV}$ equation if we substitute $\tau-t$ for $t$. Then, at the initial moment of time, $t$ $=0$, the solution (24) is a smooth function. As $t$ increases, this solution becomes narrower in space, and eventually becomes the $\delta$-function at $t=\tau$. After this it disperses again. This simple explicit example shows that short-lived anomalously large waves can appear in a nonlinear wave field that has the form of a frequency modulated train. It can be shown from the solution to the associated spectral problem (4) that, in contrast to the Korteweg-de Vries equation, the deltafunction potential has no discrete spectrum (Clarke et al, 2000). As a result, this self-similar solution does not contain any soliton-like and breather-like pulses, which implies that solitons and breathers do not necessarily play a significant role in the dispersive formation of a freak wave, if the frequency modulation of the initial wave packet is strong enough. Such frequency modulation should be close to the specific form determined by the self-similar solution (23) so 
that the wave amplification will be significant. Some theorems concerning the formation of the singularities from smooth solutions representing frequency modulated wavetrains in the framework of the generalized Korteweg-de Vries equation can be found in the work of Bona and Saut (1993). In fact, this mechanism of wave focusing from phase-modulated disturbances rapidly vanishing at infinity in the framework of the modified Korteweg-de Vries equation is qualitatively the same as for the Korteweg-de Vries equation (Pelinovsky et al., 2000) and will not discussed here in further detail.

\section{Conclusion}

In this paper we have discussed the generation of large-amplitude short-lived pulses in the framework of the modified Korteweg-de Vries equation, that plays an important role in the dynamics of meandering barotropic jets, internal waves in stratified fluids, nonlinear Alfvén waves propagating along the ambient magnetic field, and nonlinear waves in a distributed Schottky barrier diode transmission lines Breather generation from modulated wave packets is studied in the framework of the AKNS (Zakharov-Shabat) system for the modified Korteweg de Vries equation as well as in the framework of nonlinear Schrodinger equation dasymptotically derived from the modified Korteweg-de Vries for weakly nonlinear modulated wave trains. It is shown that nonlinear Schrodinger equation model overestimates the appearance of large-amplitude short-lived pulses, while in the framework of the modified Korteweg-de Vries equation breathers fission and then cease to interact. For quasi-periodic wave trains modulational instability is studied by means of the direct numerical integration of the modified Korteweg-de Vries equation. For weakly nonlinear wave packets, the nonlinear stage of the modulational instability is qualitatively the same as that described by the nonlinear Schrodinger equation; large-amplitude wave groups appear quasi-periodically. With an increase of the initial amplitude, large-amplitude groups and single pulses appear more frequently and rather randomly, while there is no recurrence to the initial state. Maximum amplification of such pulses decreases as the initial wave amplitude increases. Also, wave focusing due to difference in the group velocities of individual wave packets is noted as another mechanism for the appearance of large-amplitude pulses, where we use a self-similar solution of the $\mathrm{mKdV}$ equation is to demonstrate the effect of wave focusing. In summary, the existence of largeamplitude short-lived pulses should be considered as an important part of nonlinear wave dynamics, together with the usual long-lived solitons and breathers. 
Acknowledgements: Grants from the London Mathematical Society and Royal Society are acknowledged. EP and TT had the particular support from the RFBR (03-05-64978) and INTAS (01-0330, 03-51-3728 and 03-51-4286). 


\section{References}

Ablowitz, M.J., and Clarkson, P.A. Solitons, Nonlinear Evolution Equations and Inverse Scattering. Cambridge University Press. Cambridge, 1991.

Ablowitz, M.I., Hammack, J. Henderson, D., and Schober, C.M. Long-time dynamics of the modulational instability of deep water waves. Physica D, 2001, vol. 152-153, 416 - 433.

Bona, J.L., and Saut, J.-C. Dispersive blowup of solutions of generalized Korteweg - de Vries equations. J. Differential Equations, 1993, vol. 103, N. 1, 3-57.

Calini, A., and Schober C.M. Homoclinic chaos increases the likelihood of rogue wave formation. Physics Letters A, 2002, vol. 298, 335-349.

Clarke S., Grimshaw R., Miller P., Pelinovsky E., Talipova T. On the generation of solitons and breathers in the modified Korteweg - de Vries equation. Chaos, 2000, vol. 10, N. 2, 383 392.

Driscoll, C.F., and O'Neil, T.M. Modulational instability of cnoidal wave solutions of the modified Korteweg - de Vries equation. J. Math. Phys., 1976, vol. 17, 1196 - 1200.

Dysthe, K.B., and Trulsen, K. Note on breather type solutions of the NLS as a model for freak waves. Physica Scripta, 1999, vol. T82, 48-52.

Grimshaw R., Pelinovsky E., Talipova T. The modified Korteweg-de Vries equation in the theory of large-amplitude internal waves. Nonlinear Processes in Geophysics, 1997, vol. 4, N. 4, $237-350$.

Grimshaw, R., Pelinovsky, D., Pelinovsky, E., and Talipova, T. Wave group dynamics in weakly nonlinear long-wave models. Physica D, 2001, vol. 159, N. 1 - 2, 35 - 57.

Kakutani, T., and Ono, H. Weak non-linear hydromagnetic waves in a cold collision-free plasma. J. Phys. Soc. Japan, 1969, vol. 26, N. 5, 1305 - 1318.

Kharif C., Pelinovsky E. Physical mechanisms of the rogue wave phenomenon. European $J$ Mechanics / B - Fluid, 2003, vol. 22, N. 6, 603-634.

Kharif, C., Pelinovsky, E., Talipova, T., and Slunyaev, A. Focusing of nonlinear wave groups in deep water. JETP Letters, 2001, vol. 73, N. 4, 170 - 175.

Lamb, G.L., Elements of soliton theory. Wiley, New York, 1980.

Matveev, V.B. Positons: slowly decreasing analogues of solitons. Theoretical and Mathematical Physics, 2002, vol. 131, N. 1, 483-497. 
Mjølhus, E. Nonlinear Alfvén waves and the DNLS equation: oblique aspects. Physica Scripta, 1989, vol. 40, $227-237$.

Mjølhus, E., and Hada, T. Soliton theory of quasi-parallel MHD waves. In: Nonlinear waves and Chaos in Solar Plasmas (Eds.: T.Hada and H.Matsumoto), TERRAPUB, Tokyo, 1997, 121 $-169$.

Nycander, J., Dritschel, D.G., and Sutyrin, G.G. The dynamics of long frontal waves in the shallow-water equations. Physics Fluids, 1993, vol. A5, N. 5, 1089-1091.

Osborne, A.R., Onorato, M., and Serio, M. The nonlinear dynamics of rogue waves and holes in deep-water gravity wave train. Phys. Letters A, 2000, vol. 275, 386-393.

Parkes, E.J. The modulation of weakly non-linear dispersive waves near the marginal state of instability. J. Physics A, 1987, vol. 20, 2025-2036.

Pelinovsky, E., Talipova, T., and Kharif, C. Nonlinear dispersive mechanism of the freak wave formation in shallow water. Physica D, 2000, vol. 147, 83-94.

Ralph, E.A., and Pratt, L. Predicting eddy detachment for an equivalent barotropic thin jet. $J$. Nonlinear Science, 1994, vol. 4, N. 4, 355-374.

Slunyaev, A., Kharif, C., Pelinovsky, E., and Talipova, T. Nonlinear wave focusing on water of finite depth. Physica D, 2002, vol. 173, 77-96.

Talipova T., Pelinovsky E., Lamb K., Grimshaw R., Holloway P. Cubic nonlinearity effects in the propagation of intense internal waves. Doklady Earth Sciences, 1999, vol. 365, N. 2, 241 244.

Wazwaz, A-M., A study on compacton-like solutions for the modified KdV and fifth-order KdV-like equations. Applied Mathematics and Computation, 2003, vol. 147, 439-447.

Zieger, V., Dinkel, J., Setzer, C., and Lonngren, K. On the propagation of nonlinear solitary waves in a distributed Schottky barrier diode transmission line. Chaos, Solitons and Fractals, 2001, vol. 12, 1719-1728. 


\section{Figure Captions}

Fig. 1. Wave packet evolution for $k=10$ and $a=2(\mathrm{mKdV}-$ left, NLS - right)

Fig. 2. Wave packet evolution for $k=4$ and $a=4$ (mKdV - left, NLS - right)

Fig. 3. Breather fission on large times (mKdV model)

Fig. 4. Wave evolution in the non-focusing case

Fig. 5. Wave trajectories for the initial amplitude $a=0.08$

Fig. 6. The same as in Fig. 2, but for $t<600$

Fig. 7. Snapshots of the wave field with the initial amplitude $a=0.08$

Fig. 8. The maximum value of the wave amplitude in the domain versus time for the initial amplitude $a=0.08$

Fig. 9. Wave trajectories for the initial amplitude $a=0.16$

Fig. 10. Snapshots of the wave field with the initial amplitude $a=0.16$

Fig. 11. The maximum value of the wave amplitude in the domain versus time for the initial amplitude $a=0.16$

Fig. 12. The wave trajectories for the initial amplitude $a=0.32$

Fig. 13. The same as on Figure 12, but for the wave amplitudes exceeding 0.44

Fig. 14. Snapshots of the wave field with the initial amplitude $a=0.32$

Fig. 15. The maximum wave amplitude in the domain versus time for the initial amplitude $a=$ 0.32 

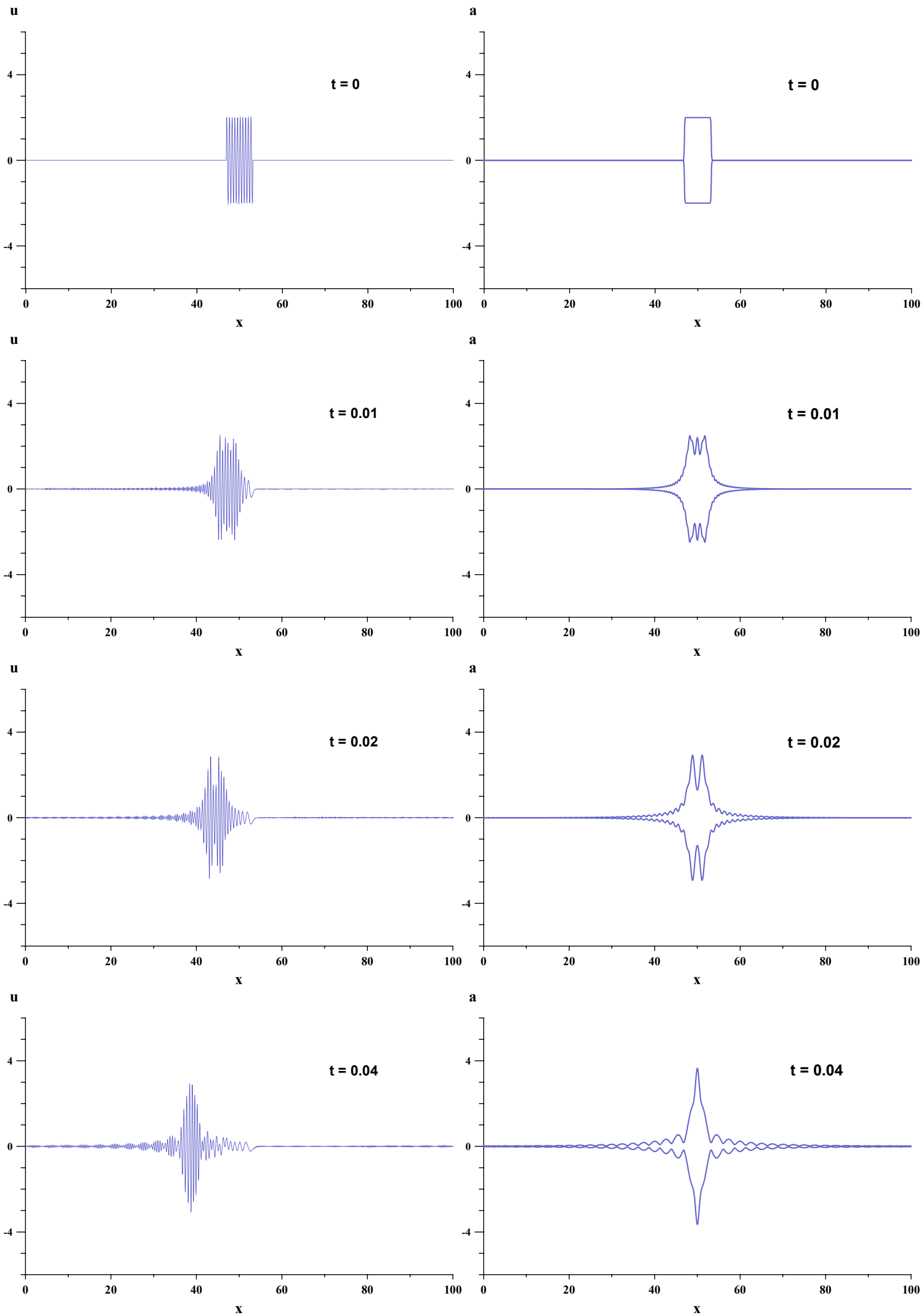

Fig. 1. Wave packet evolution for $k=10$ and $a=2(\mathrm{mKdV}-$ left, NLS - right) 

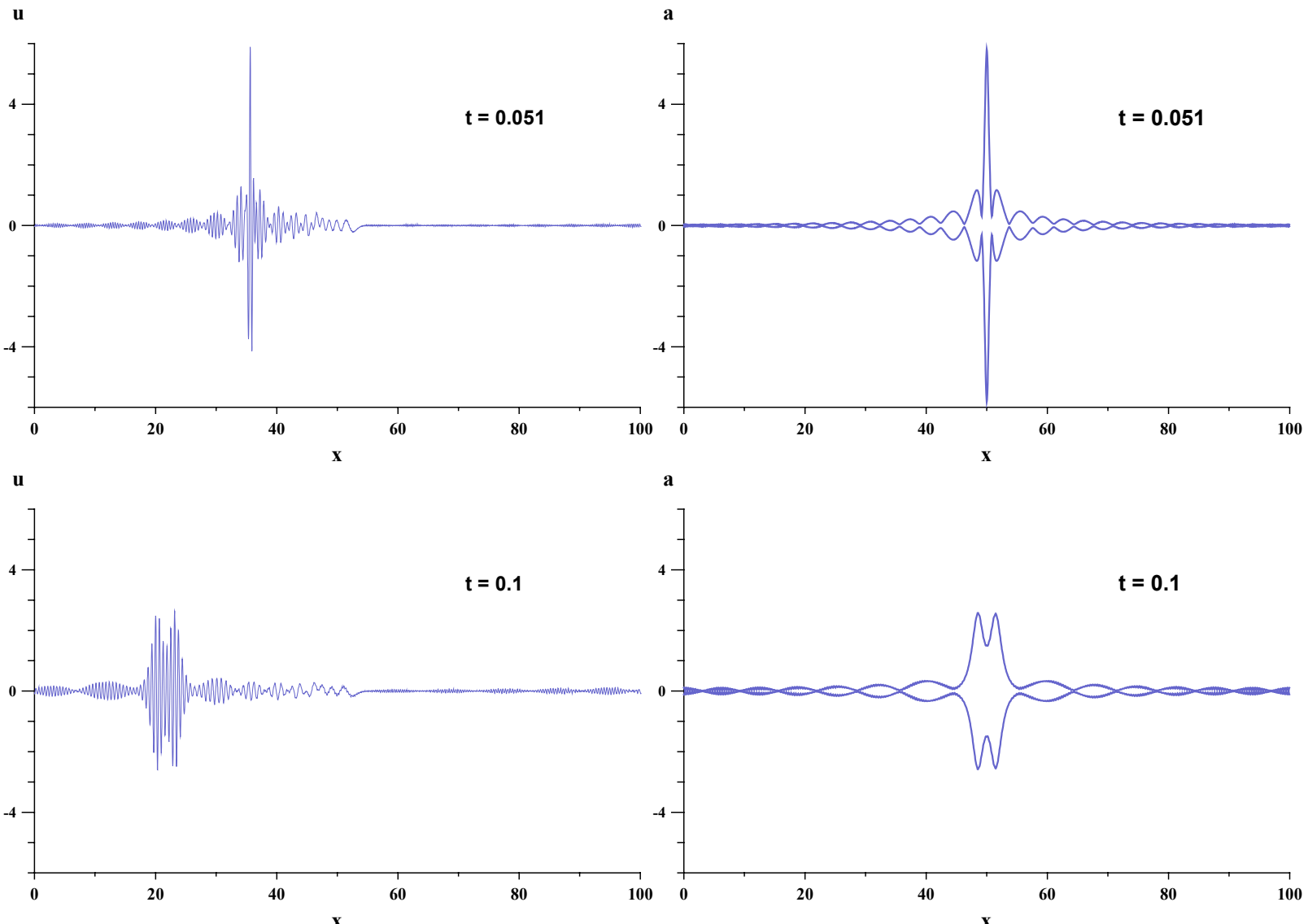

Fig. 1. Wave packet evolution for $k=10$ and $a=2(\mathrm{mKdV}-$ left, NLS - right $)$ - continued
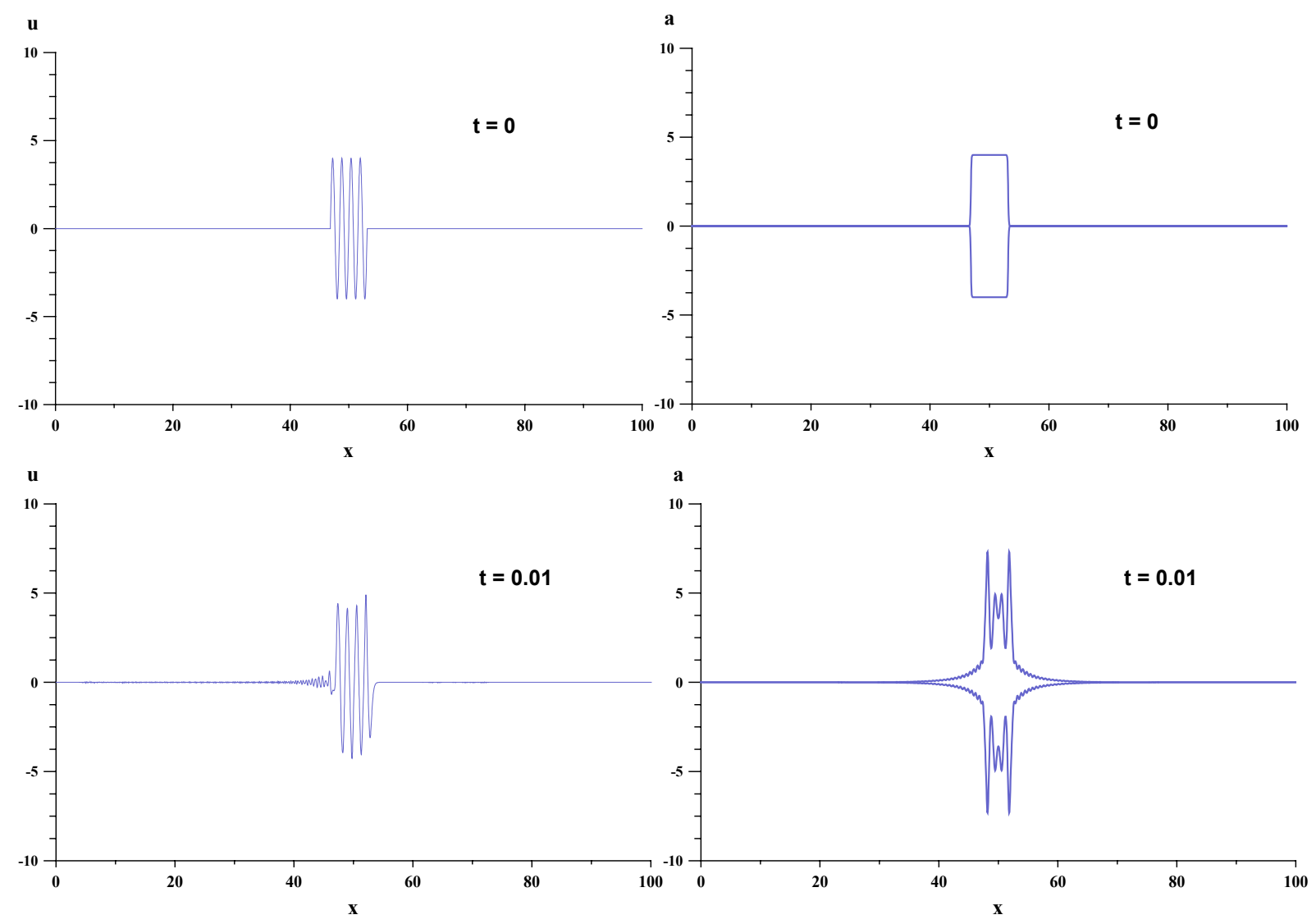

Fig. 2. Wave packet evolution for $k=4$ and $a=4(\mathrm{mKdV}-$ left, NLS - right) 

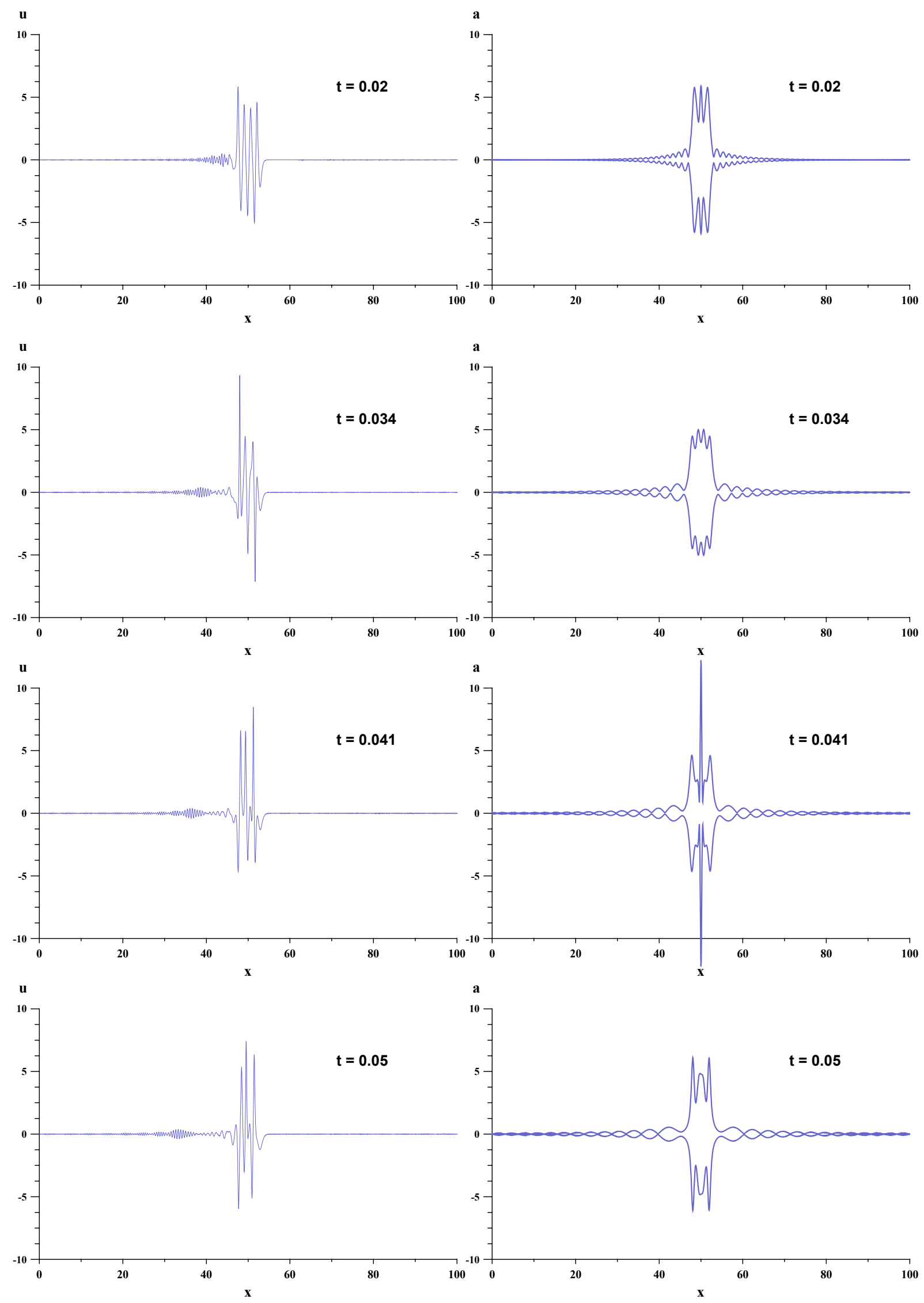

Fig. 2. Wave packet evolution for $k=4$ and $a=4(\mathrm{mKdV}$ - left, NLS - right) - continued 


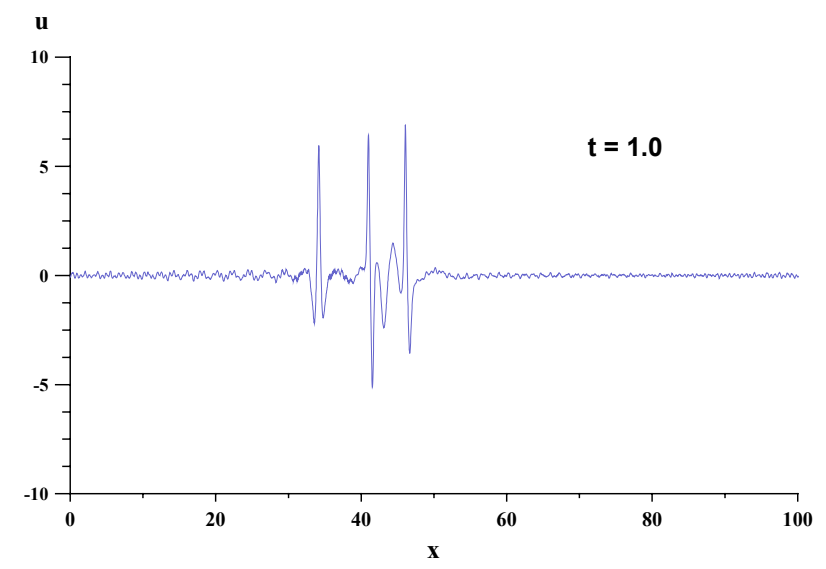

Fig. 3. Breather fission on large times (mKdV model)
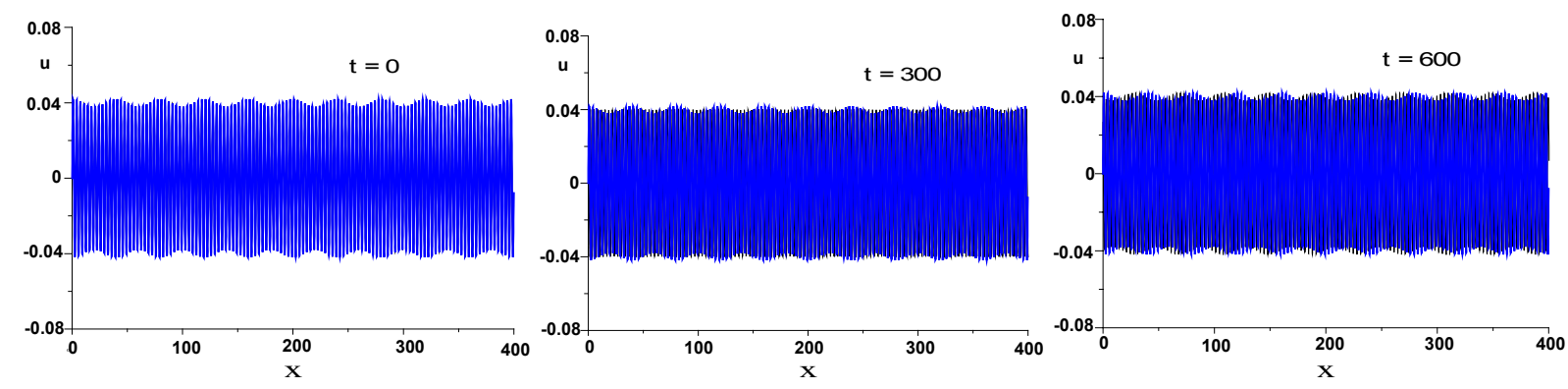

Fig. 4. Wave evolution in the non-focusing case 


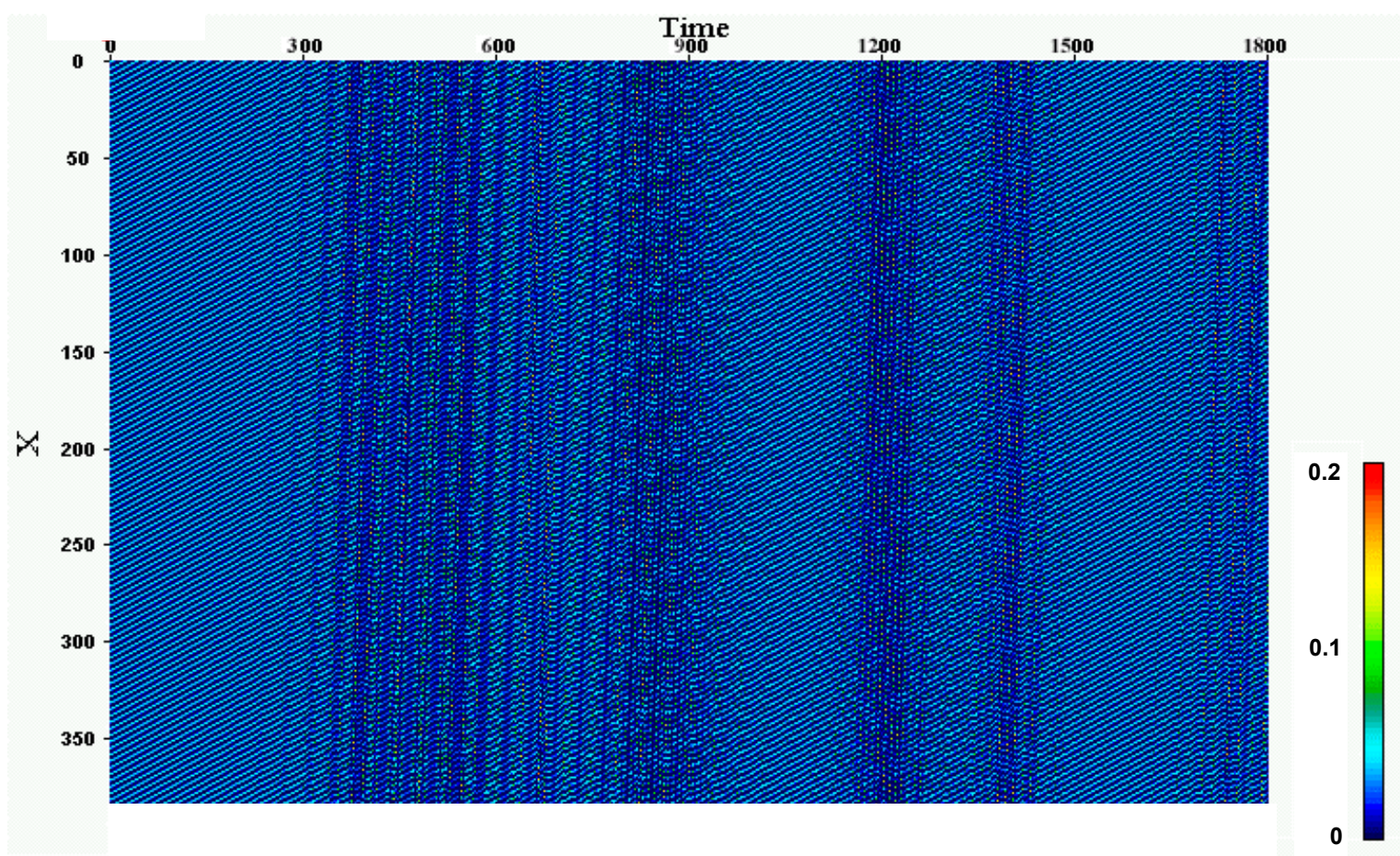

Fig. 5. Wave trajectories for the initial amplitude $a=0.08$

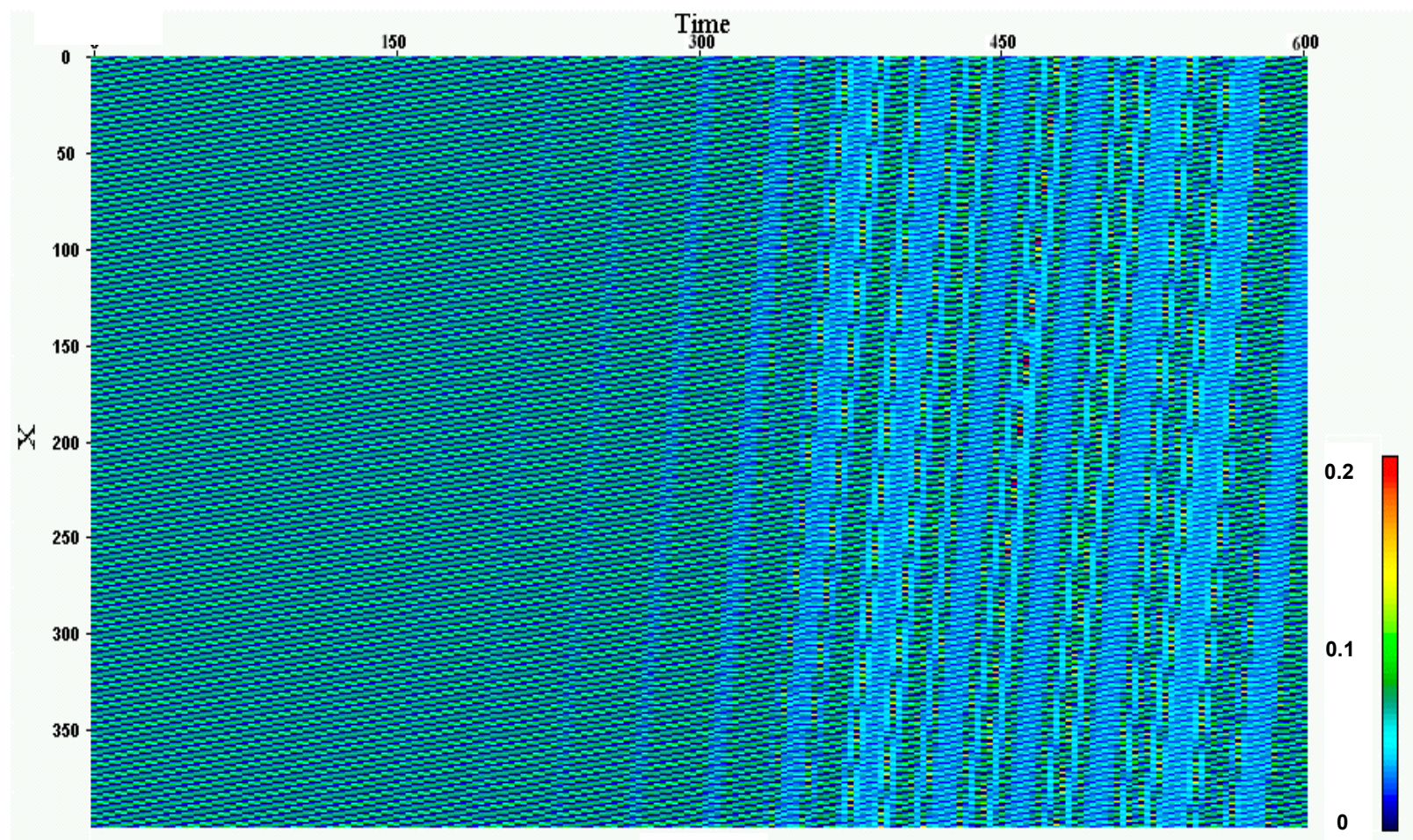

Fig. 6. The same as in Fig. 2, but for $t<600$ 

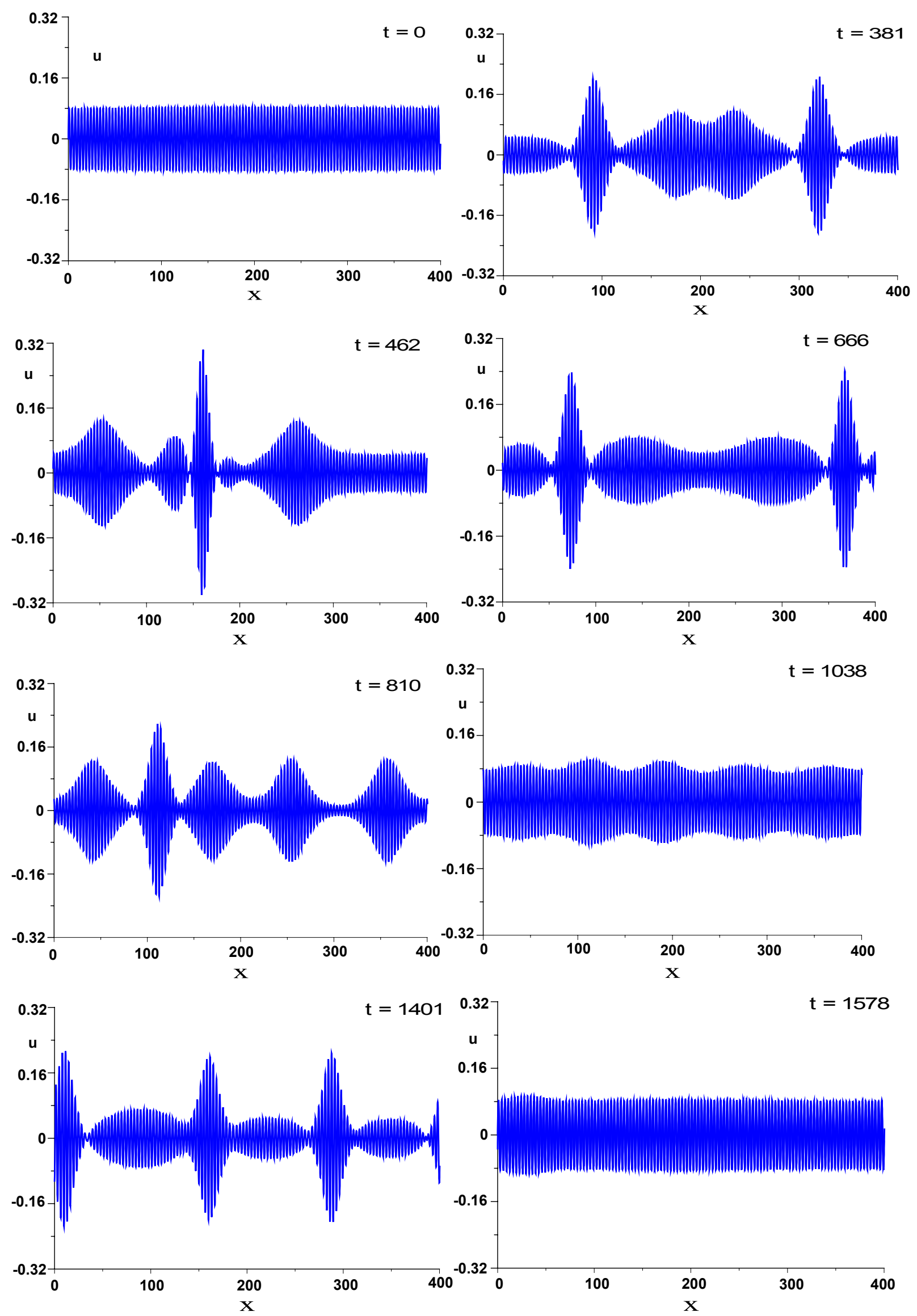

Fig. 7. Snapshots of the wave field with the initial amplitude $a=0.08$ 


\section{$a_{\max }$}

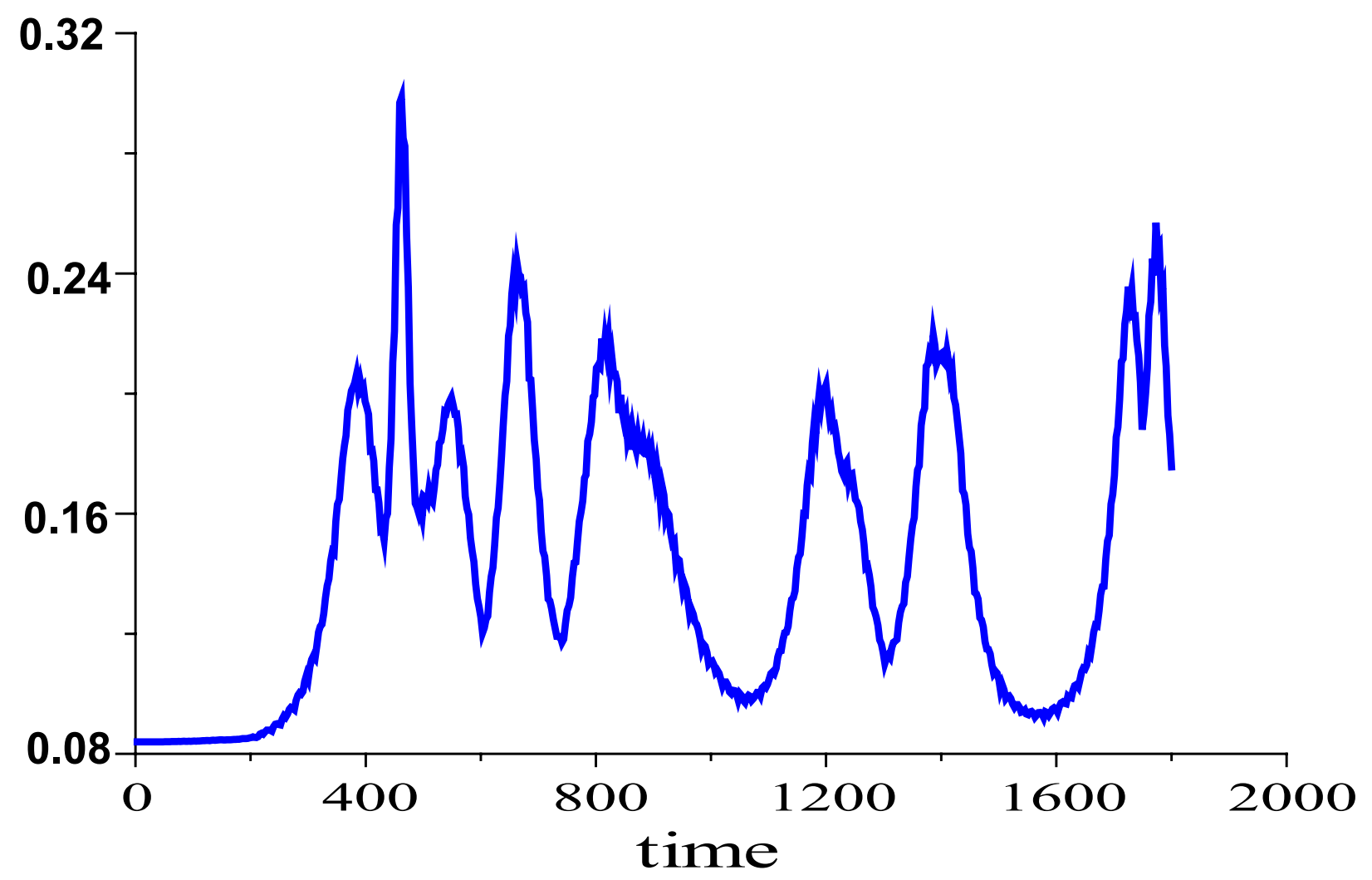

Fig. 8. The maximum value of the wave amplitude in the domain versus time for the initial amplitude $a=$ 0.08

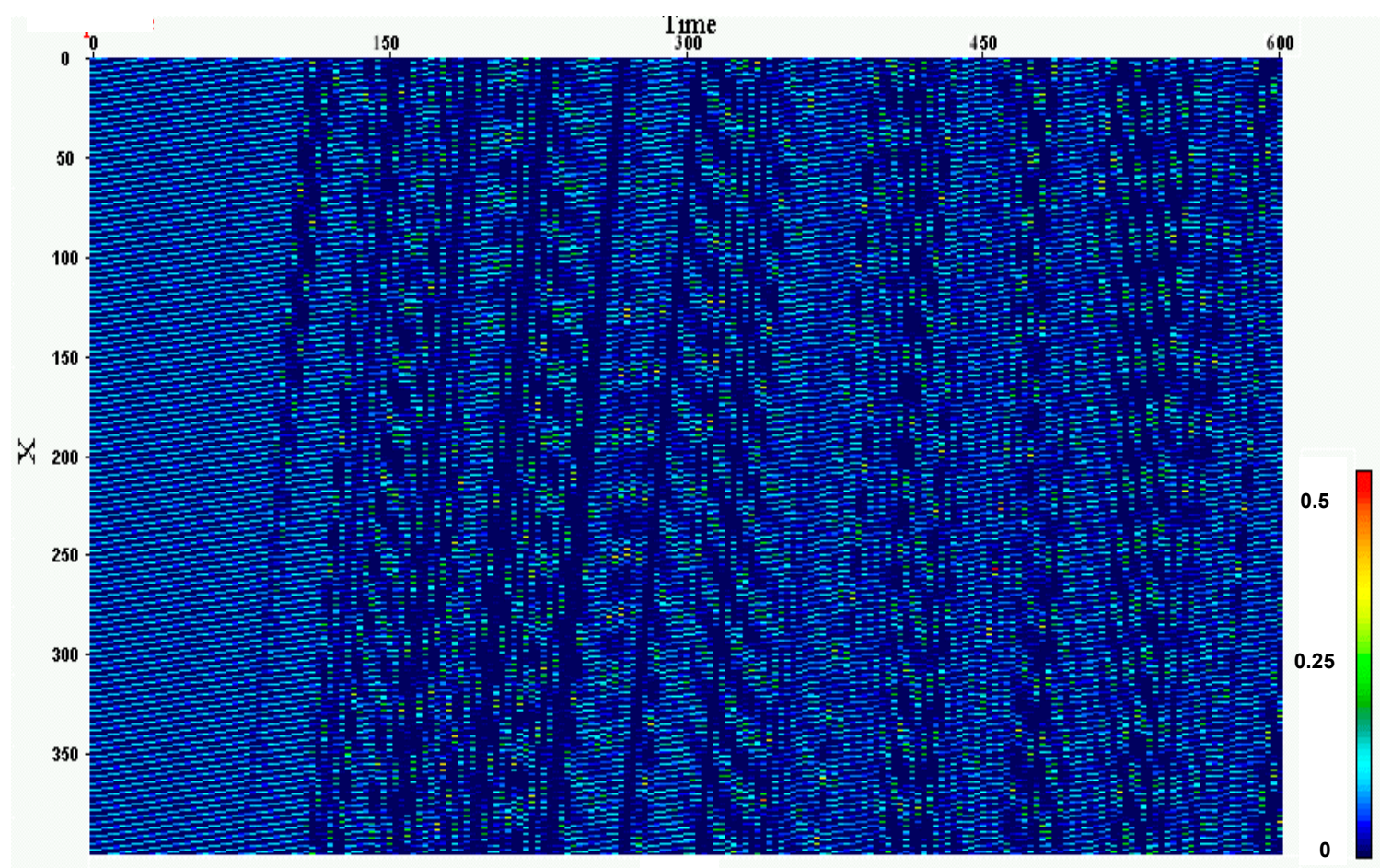

Fig. 9. Wave trajectories for the initial amplitude $a=0.16$ 

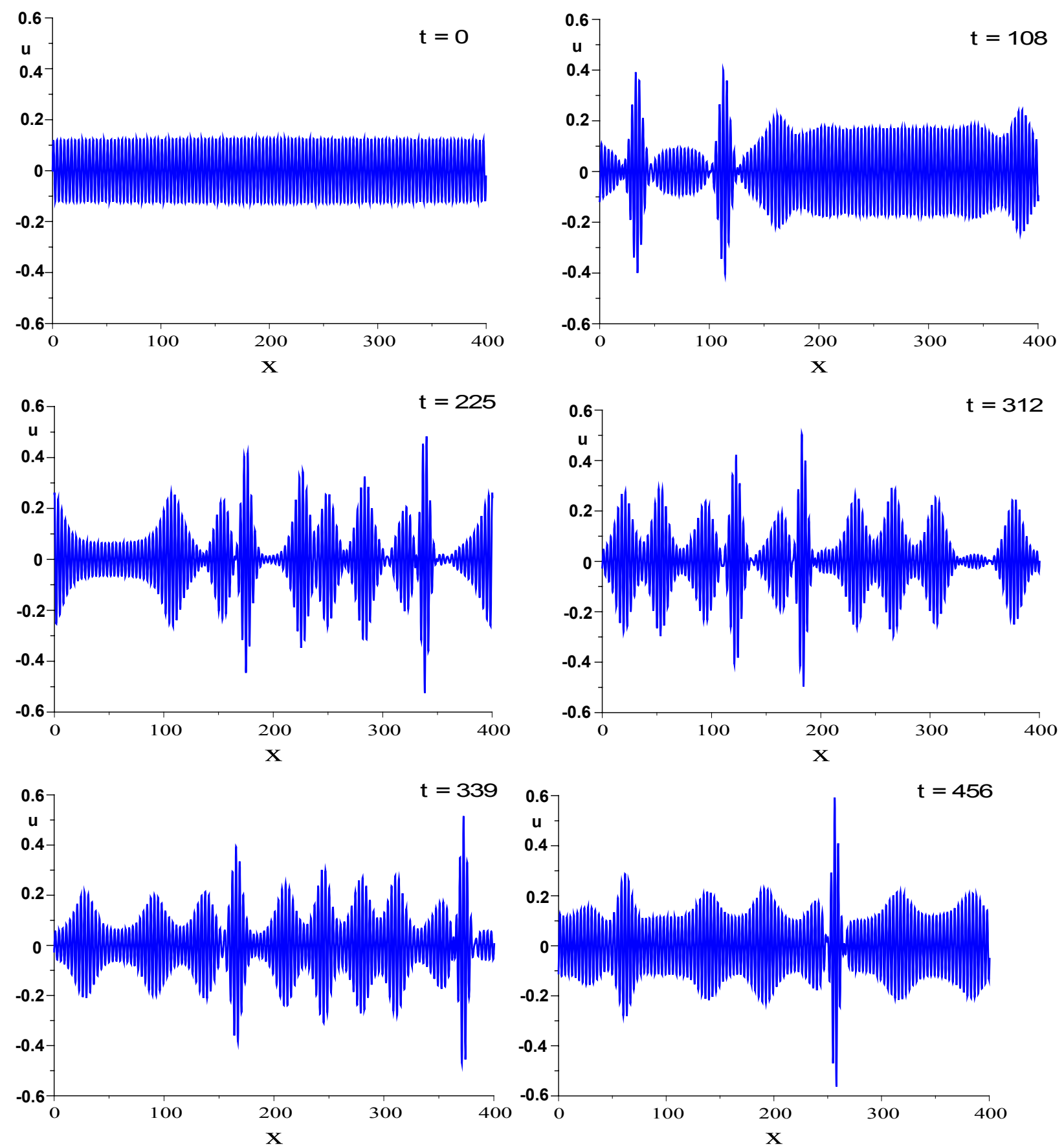

Fig. 10. Snapshots of the wave field with the initial amplitude $a=0.16$ 


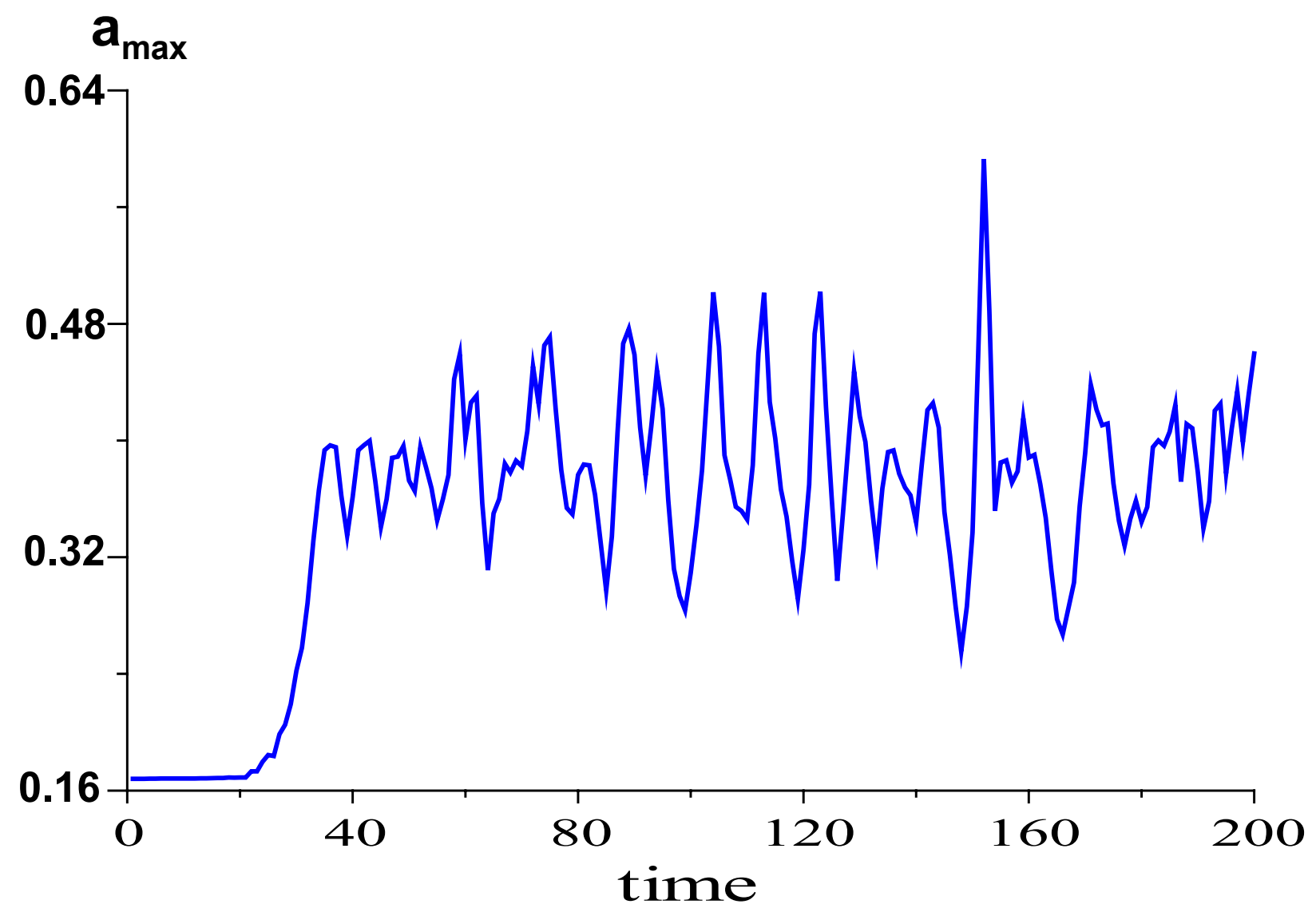

Fig. 11. The maximum value of the wave amplitude in the domain versus time for the initial amplitude $a=0.16$

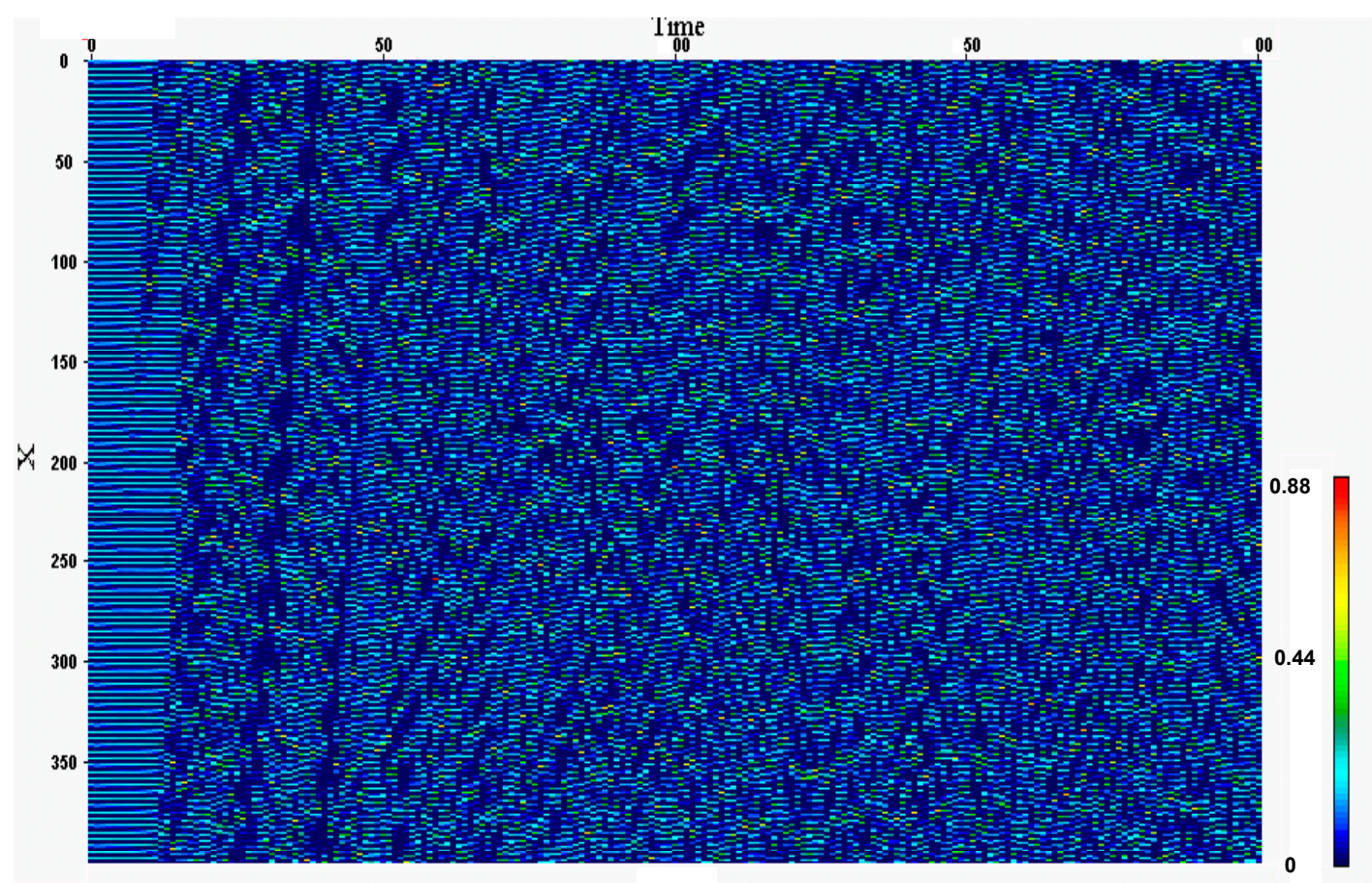

Fig. 12. The wave trajectories for the initial amplitude $a=0.32$ 


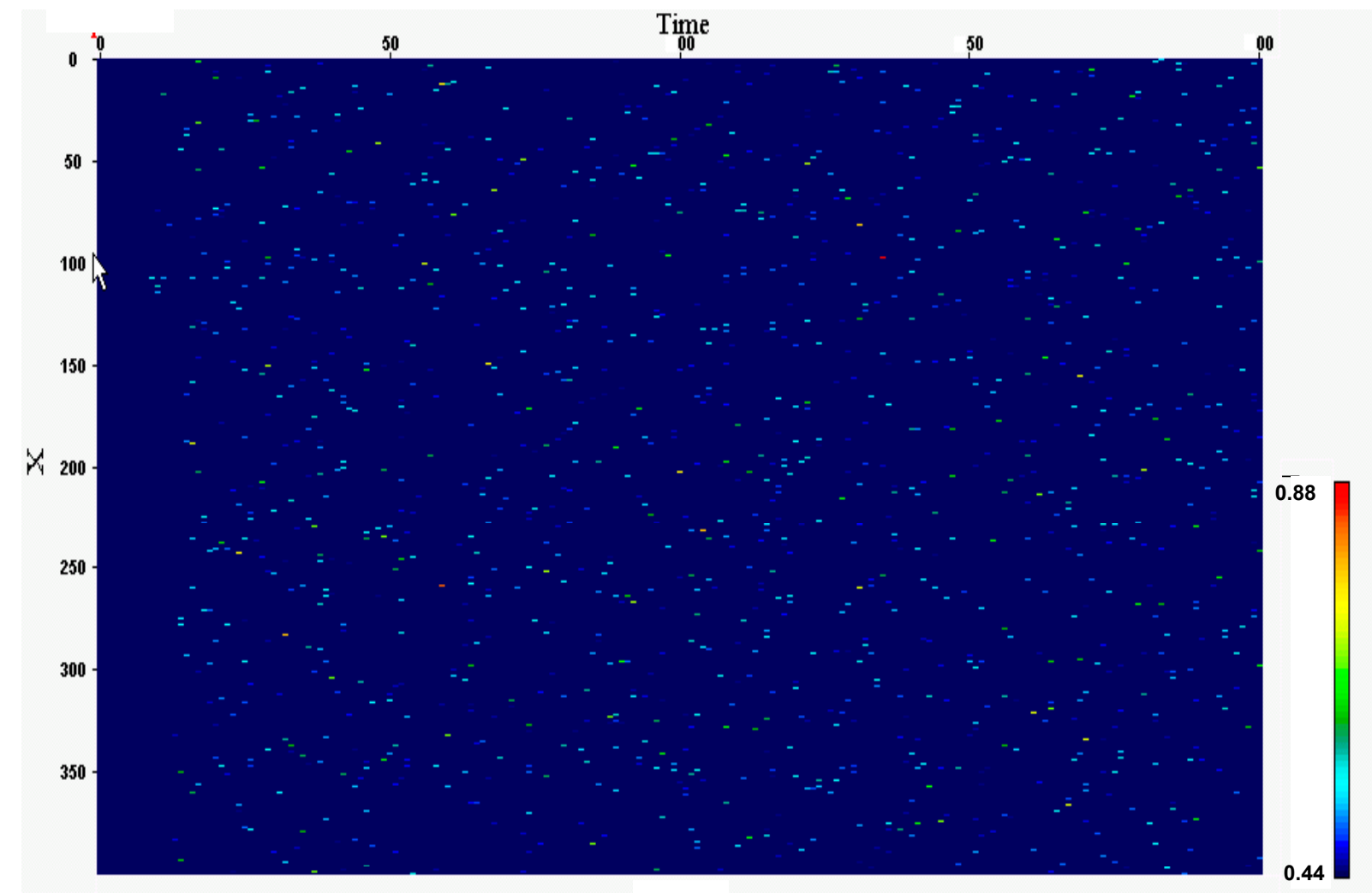

Fig. 13. The same as on Figure 12, but for the wave amplitudes exceeding 0.44 

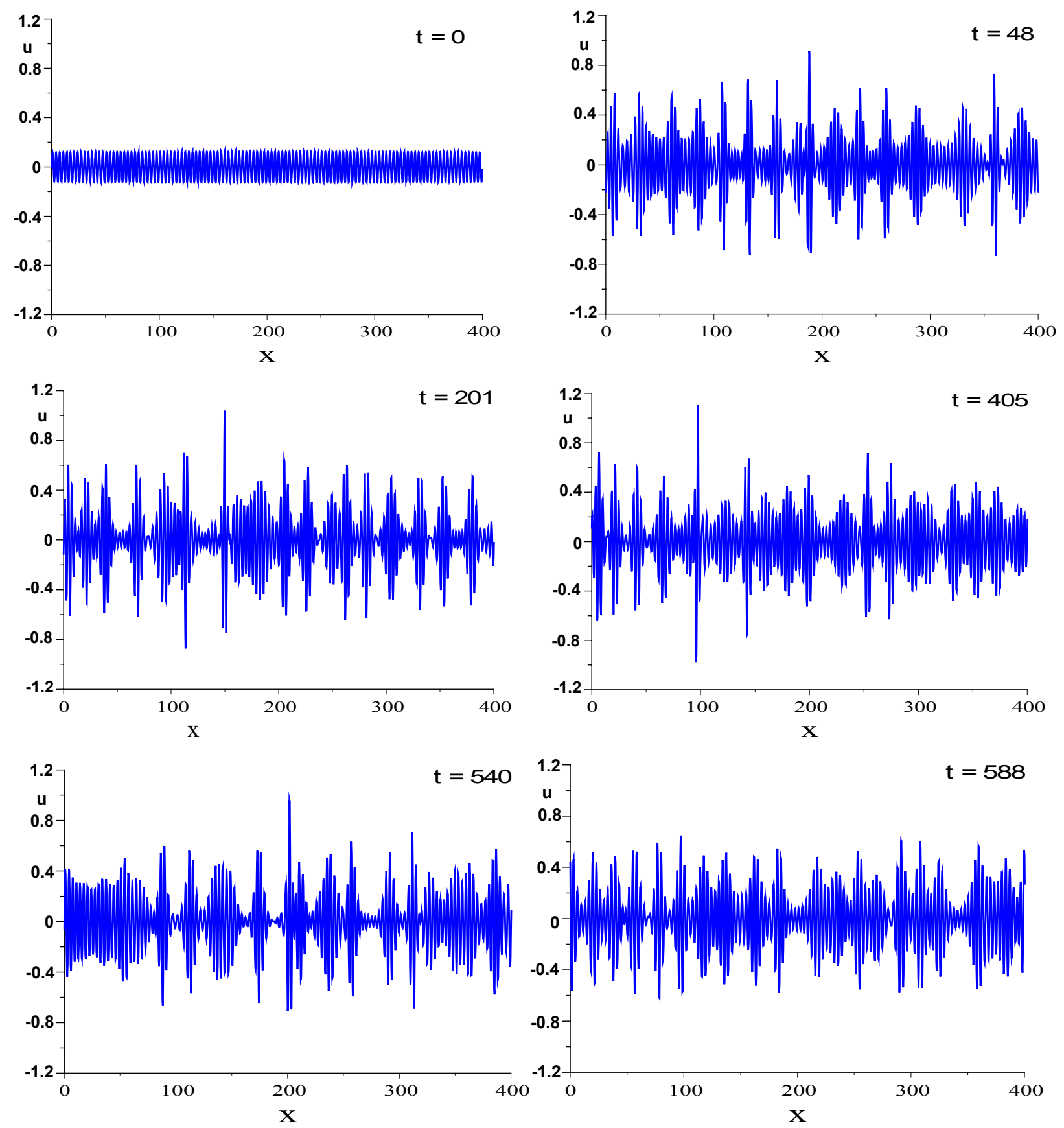

Fig. 14. Snapshots of the wave field with the initial amplitude $a=0.32$ 


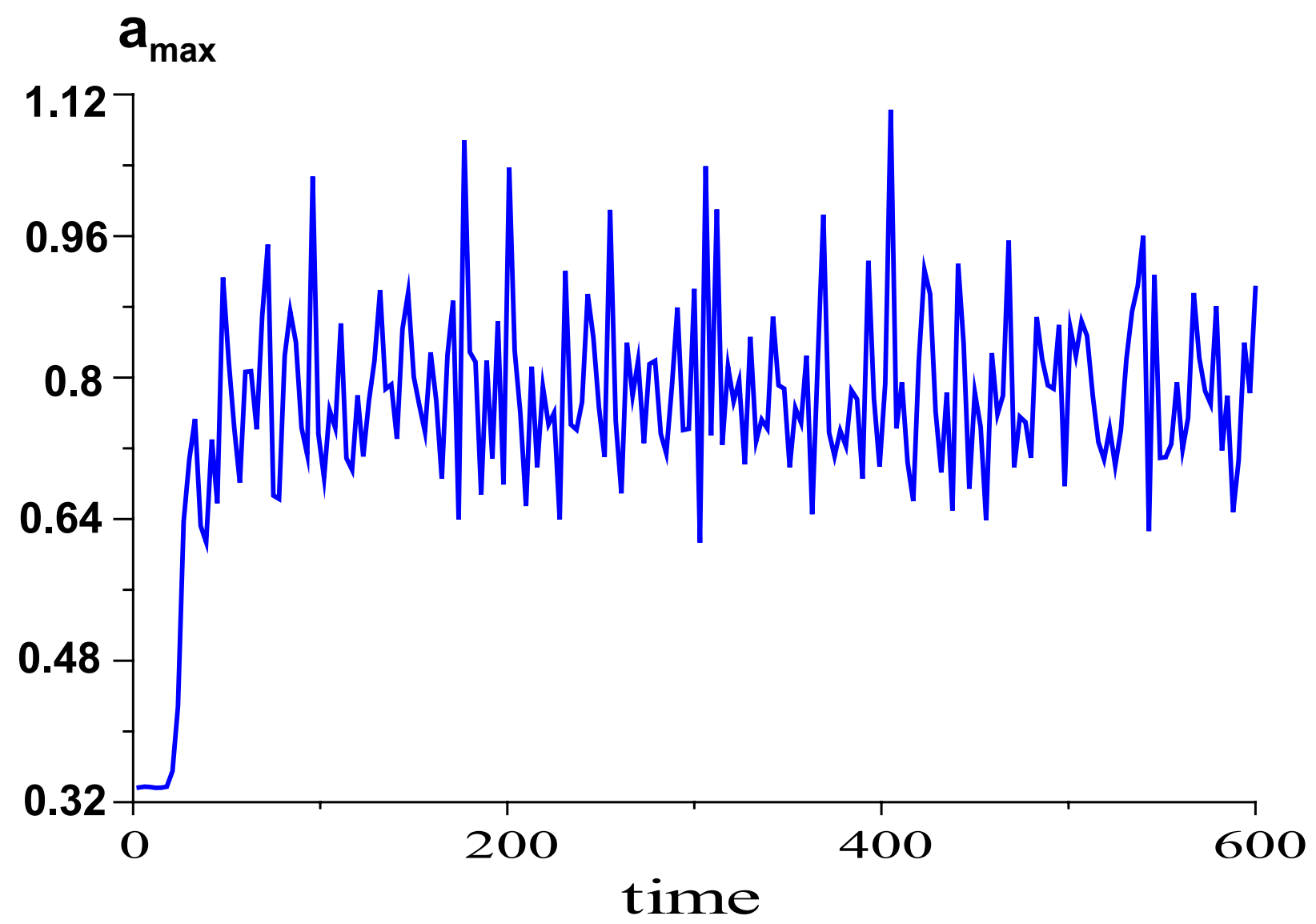

Fig. 15. The maximum wave amplitude in the domain versus time for the initial amplitude $a=0.32$ 
Figures 5, 6, 9, 12, 13 as white-black
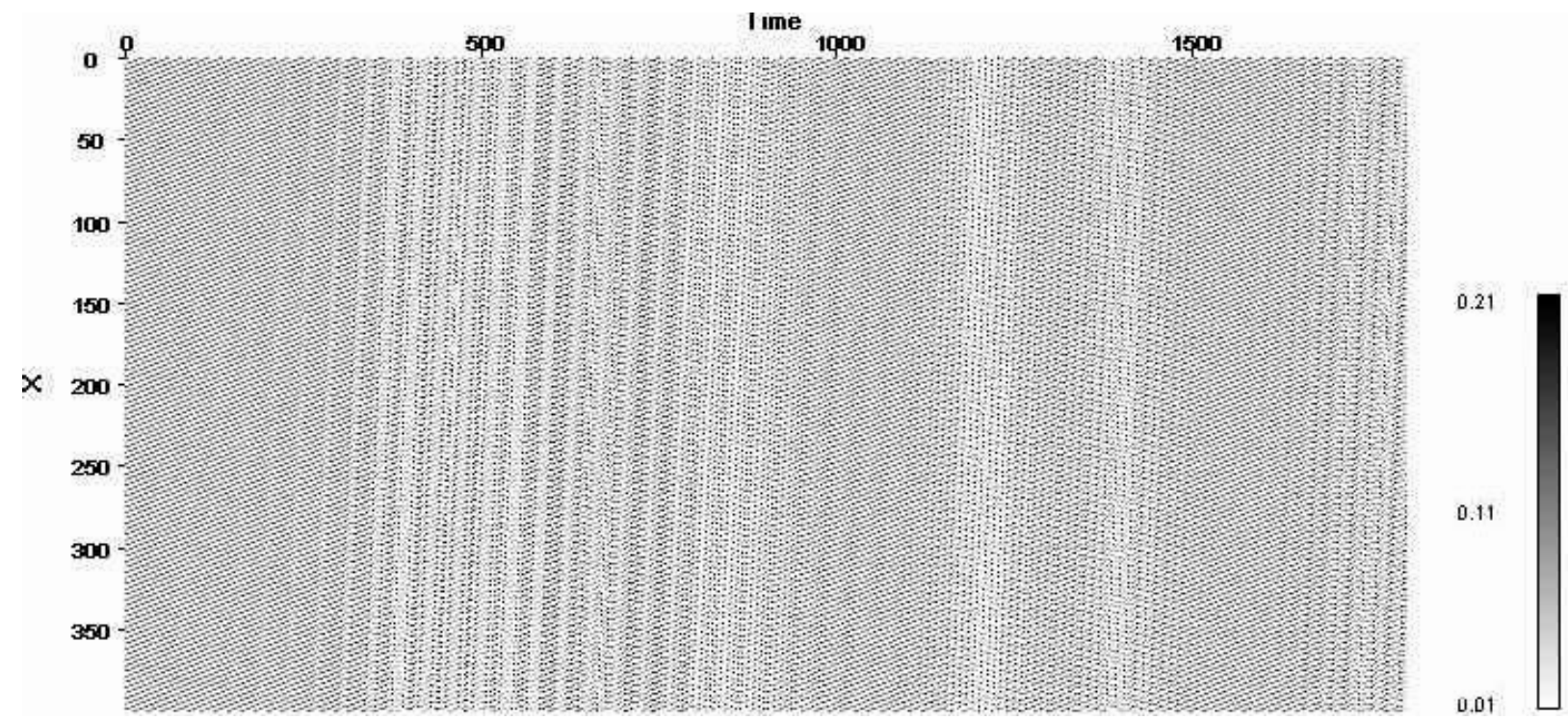

Fig.5.
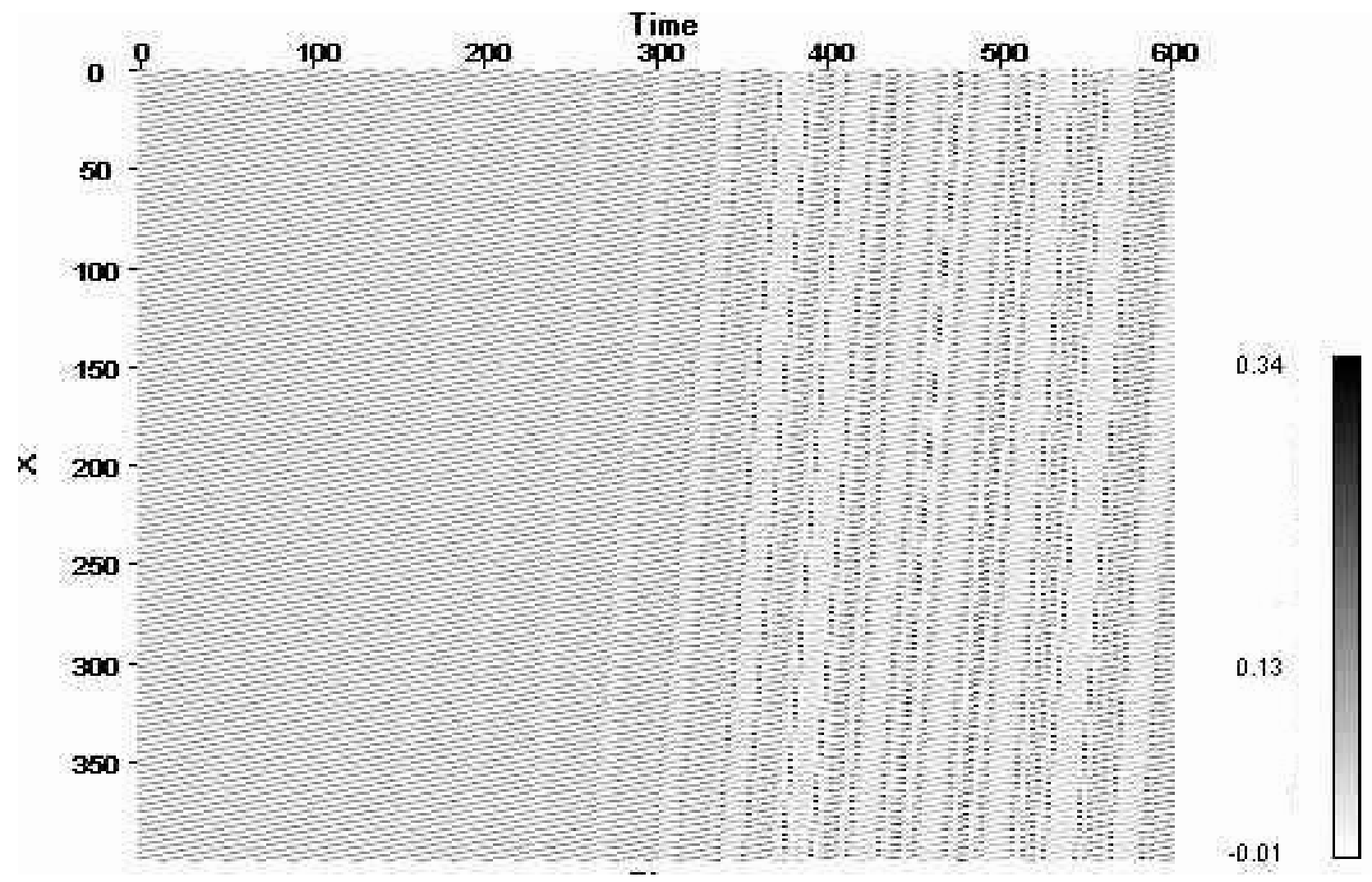

Fig.6. 


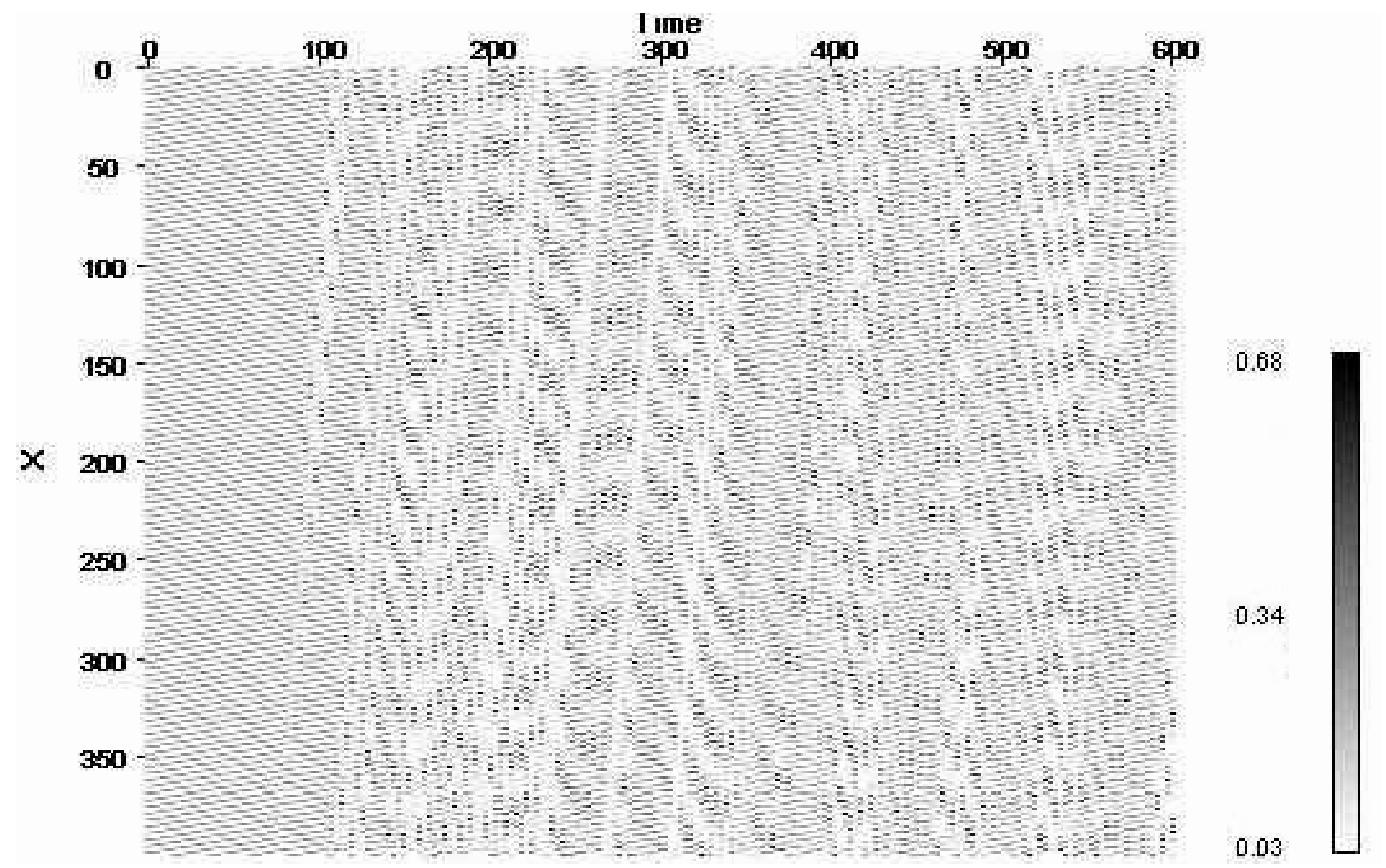

Fig.9.

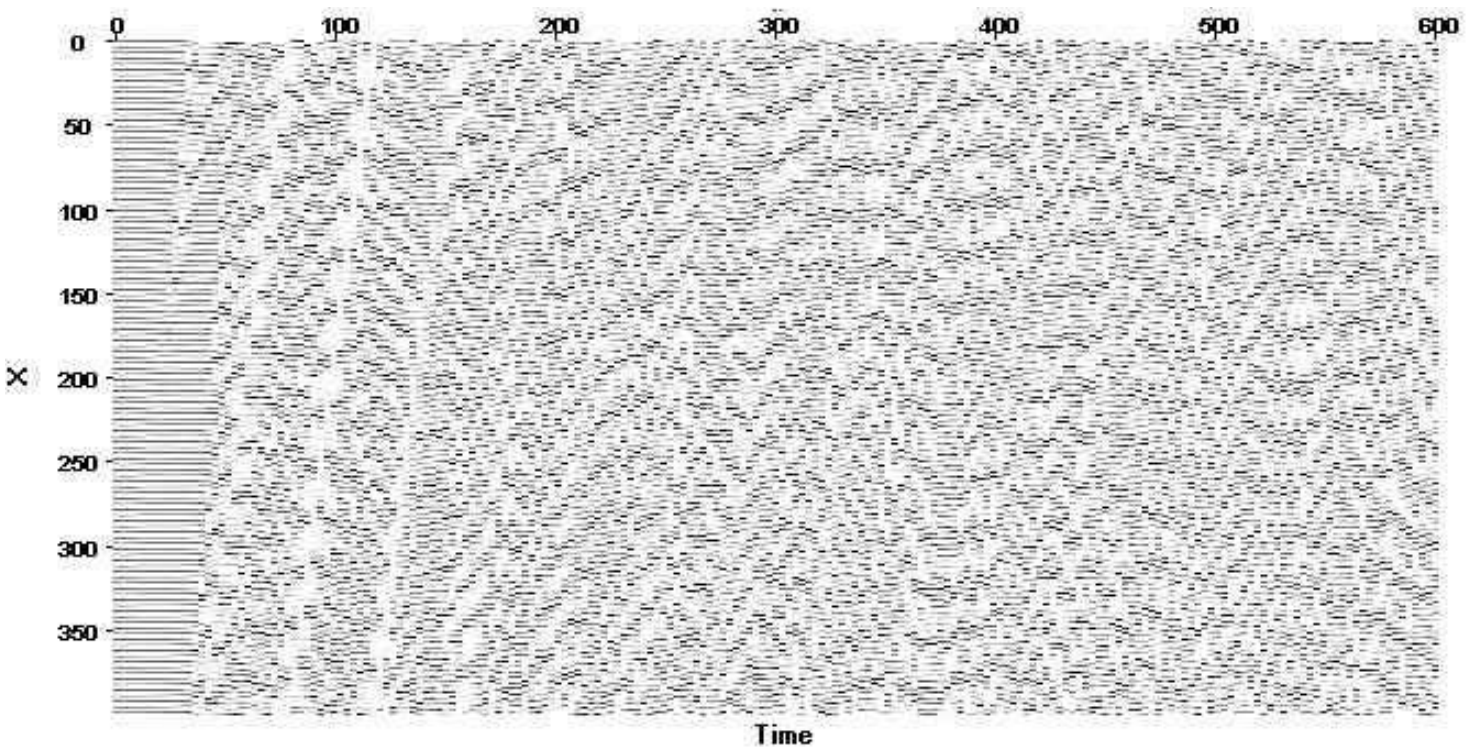

Fig. 12. 


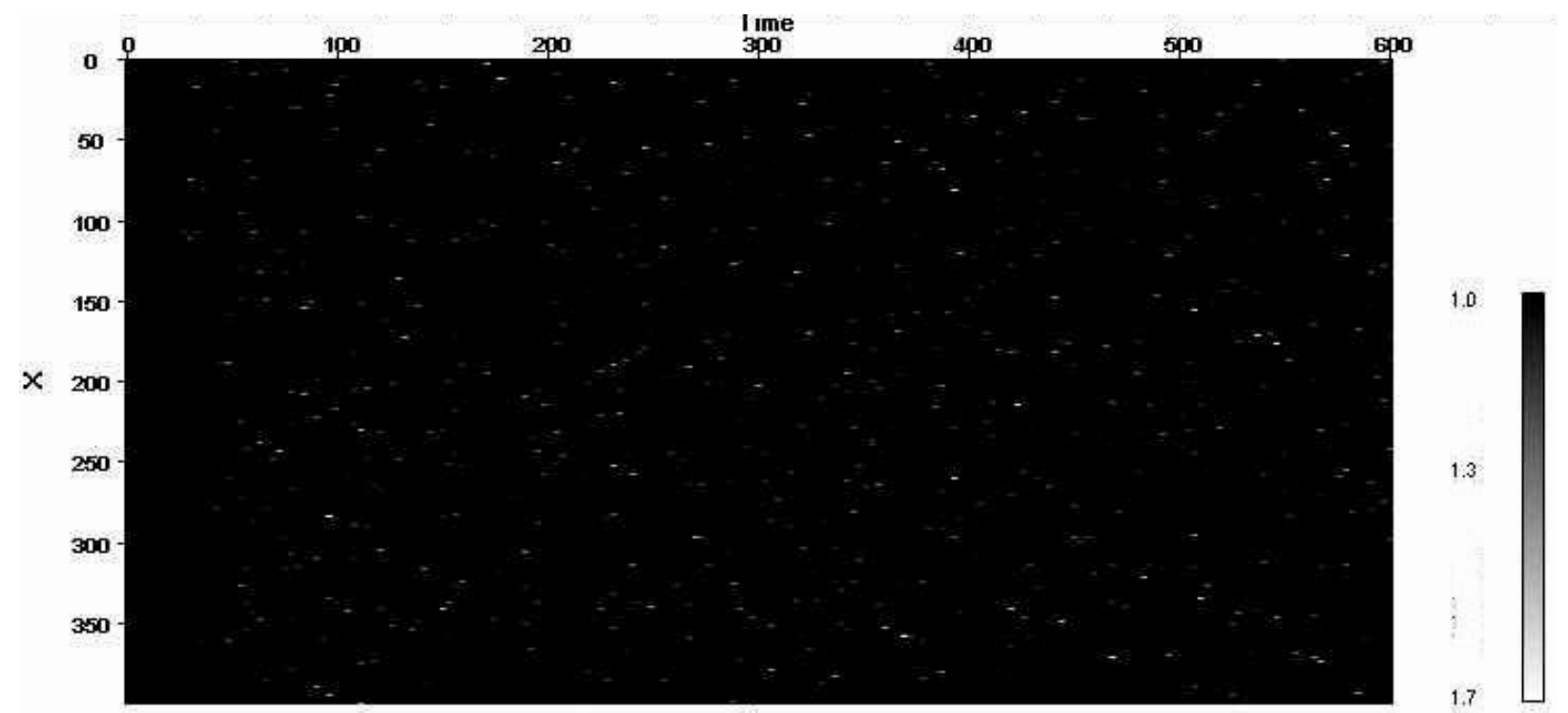

Fig.13 